\title{
AFRL-RI-RS-TR-2015-032
}

\section{DISCRETE RICCI FLOW IN HIGHER DIMENSIONS}

FLORIDA ATLANTIC UNIVERSITY

FEBRUARY 2015

FINAL TECHNICAL REPORT

STINFO COPY

\section{AIR FORCE RESEARCH LABORATORY INFORMATION DIRECTORATE}




\section{NOTICE AND SIGNATURE PAGE}

Using Government drawings, specifications, or other data included in this document for any purpose other than Government procurement does not in any way obligate the U.S. Government. The fact that the Government formulated or supplied the drawings, specifications, or other data does not license the holder or any other person or corporation; or convey any rights or permission to manufacture, use, or sell any patented invention that may relate to them.

This report was cleared for public release by the $88^{\text {th }}$ ABW, Wright-Patterson AFB Public Affairs Office and is available to the general public, including foreign nationals. Copies may be obtained from the Defense Technical Information Center (DTIC) (http://www.dtic.mil).

AFRL-RI-RS-TR-2015-032 HAS BEEN REVIEWED AND IS APPROVED FOR PUBLICATION IN ACCORDANCE WITH ASSIGNED DISTRIBUTION STATEMENT.

FOR THE DIRECTOR:

/ S /

PAUL ALSING

Work Unit Manager
/ S /

MARK H. LINDERMAN

Technical Advisor, Computing

\& Communications Division

Information Directorate

This report is published in the interest of scientific and technical information exchange, and its publication does not constitute the Government's approval or disapproval of its ideas or findings. 


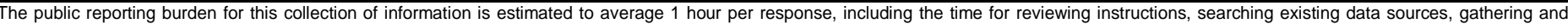

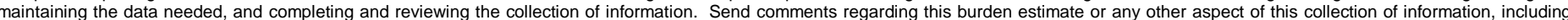

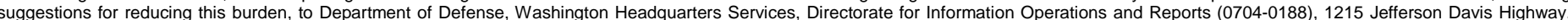

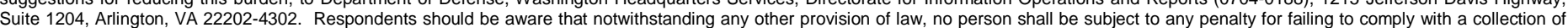
Suite 1204, Arlington, VA 22202-4302. Respondents should be aware

PLEASE DO NOT RETURN YOUR FORM TO THE ABOVE ADDRESS.

\begin{tabular}{|c|c|r}
\hline 1. REPORT DATE $(D D-M M-Y Y Y Y)$ & 3. REPORT TYPE & SES COVERED (FrOm - To) \\
FEB 2015 & FINAL TECHNICAL REPORT & SEP - SEP 2014 \\
\hline
\end{tabular}

4. TITLE AND SUBTITLE

DISCRETE RICCI FLOW IN HIGHER DIMENSIONS

\section{5a. CONTRACT NUMBER}

FA8750-11-2-0089

5b. GRANT NUMBER

N/A

5c. PROGRAM ELEMENT NUMBER

$62788 \mathrm{~F}$
6. AUTHOR(S)

Warner A. Miller
T2GT

5e. TASK NUMBER

UF

5f. WORK UNIT NUMBER

$\mathrm{AU}$
7. PERFORMING ORGANIZATION NAME(S) AND ADDRESS(ES)

Department of Physics, Florida Atlantic University

777 Glades Road

Boca Raton, FL 33431
8. PERFORMING ORGANIZATION REPORT NUMBER
9. SPONSORING/MONITORING AGENCY NAME(S) AND ADDRESS(ES)

Air Force Research Laboratory/RITA

525 Brooks Road

Rome NY 13441-4505
10. SPONSOR/MONITOR'S ACRONYM(S)

AFRL/RI

11. SPONSOR/MONITOR'S REPORT NUMBER

AFRL-RI-RS-TR-2015-032

\section{DISTRIBUTION AVAILABILITY STATEMENT}

Approved for Public Release; Distribution Unlimited. PA\# 88ABW-2015-0661

Date Cleared: 24 FEB 2015

\section{SUPPLEMENTARY NOTES}

\section{ABSTRACT}

The objective of this research to develop an efficient and justifiable algorithm to geometrize a given closed 3-manifold, and to show how its topologic characterization can be applied to complex networks. Hamilton's Ricci flow (RF) was developed in order to geometrize such 3-manifolds. The geometrization theorem (GT) states that each prime 3-manifold is either geometric or its simple pieces are geometric. The continuum approach is not numerically practical. Accordingly, we developed a discrete piecewise linear (PL) version of Hamilton's RF. It is the first dimensionally agnostic generalization of RF for PL geometries. We refer to our approach as simplicial Ricci flow (SRF). For a broad class of examples, the SRF equations reproduced the continuum RF. SRF provides an efficient approach to the 3-manifold recognition problem. Student S. Ray applied SRF to implement the 1916 Weyl isometric embedding problem. His results are being used to develop a discrete quasi-local measure of congestion in networks -- a possible filtration parameter to guide network reconfiguration.

\section{SUBJECT TERMS}

Ricci Flow, Simplicial Ricci Flow, Discrete Exterior Calculus, Regge Calculus, Piecewise Linear Complex

\begin{tabular}{|c|c|c|c|c|}
\hline \multicolumn{2}{|l|}{ 16. SECURITY CLASSIFICATION OF: } & $\begin{array}{l}\text { 17. LIMITATION OF } \\
\text { ABSTRACT }\end{array}$ & $\begin{array}{c}\text { 18. NUMBER } \\
\text { OF PAGES }\end{array}$ \\
\cline { 1 - 2 } $\begin{array}{c}\text { a. REPORT } \\
U\end{array}$ & $\begin{array}{c}\text { b. ABSTRACT } \\
U\end{array}$ & $\begin{array}{c}\text { c. THIS PAGE } \\
\text { U }\end{array}$ & SAR & 38 \\
\hline
\end{tabular}

PAUL M. ALSING

19b. TELEPHONE NUMBER (Include area code) $315-330-4960$ 


\section{Contents}

1 SUMMARY 1

2 INTRODUCTION $\quad 1$

3 METHODS, ASSUMPTIONS AND PROCEDURES 3

3.1 Five Metrics and Milestones and Accomplishments. . . . . . . . . . . . . . 3

3.2 Publications Under this Effort . . . . . . . . . . . . . . . . . . . 4

4 RESULTS AND DISCUSSION

4.1 Ricci Flow on Simplicial Geometries: the SRF Equations . . . . . . . . . . . . 5

4.2 Tetrad Formulation of Hamilton's Ricci Flow Equation _ . . . . . . . . . . . . 6

4.3 Curvature of a Piecewise-Flat $d$-Dimensional Simplicial Geometry: the $R m$ and

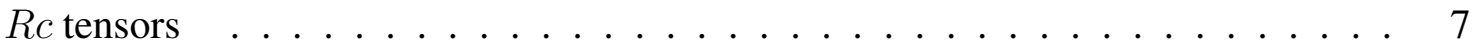

4.4 The Simplicial Ricci Flow Equation . . . . . . . . . . . . . . . . . . . . 19

4.5 Benchmarking the SRF equations on 3-manifolds . . . . . . . . . . . . 21

4.6 SRF: Singularities, Surgery, Stability and the Simplicial Diffeomorphisms . . . . . 24

4.7 Adiabatic Isometric Map: Applying SRF to Weyl's 1916 Isometric Embedding Theorem for Surfaces . . . . . . . . . . . . . . . . . . . . 26

5 CONCLUSIONS $\quad 27$

6 REFERENCES 29

7 LIST OF SYMBOLS, ABBREVIATIONS AND ACRONYMS 33 


\section{List of Figures}

1 The five hybrid 4-dimensional polytopes. . . . . . . . . . . . . . . 9

2 We illustrate here the lattice geometry used to define the $R c$ tensor for a 3-dimensional simplicial lattice. . . . . . . . . . . . . . . . . . . . 15

3 An illustration of the various geometric elements that were used to define the $R c_{\lambda}$ in 4-dimensions. . . . . . . . . . . . . . . . . . . 17

4 The geometric environs of an edge in a 4-dimensional simplicial lattice (left), one of its six hybrid blocks (center) and the edge-based hybrid area (right). . . . . . . 19

5 A 2-dimensional rendering of the simplicial double-lobed model. . . . . . . . . . . 21

6 The 2-dimensional triangulated region around the vertex, $\mathcal{O}$, on a spherical crosssection of the axisymmetric geometry. . . . . . . . . . . . . . . 23

7 This figure visualizes the three steps of the AIM algorithm. . . . . . . . . . . . 27 


\section{SUMMARY}

The objective of this research to develop an efficient and justifiable algorithm to geometrize a given closed 3-manifold, and to show how its topologic characterization can be applied to complex networks. Hamilton's Ricci flow (RF) was developed in order to geometrize such 3-manifolds. The geometrization theorem (GT) states that each prime 3-manifold is either geometric or its simple pieces are geometric. The continuum approach is not numerically practical. Accordingly, we developed a discrete piecewise linear (PL) version of Hamilton's RF. It is the first dimensionally agnostic generalization of RF for PL geometries. We refer to our approach as simplicial Ricci flow (SRF). For a broad class of examples, the SRF equations reproduced the continuum RF. SRF provides an efficient approach to the 3-manifold recognition problem. A $\mathrm{Ph}$. $\mathrm{D}$. student funded under this effort (S. Ray) applied SRF to implement the 1916 Weyl isometric embedding problem. His results are being used to develop a discrete quasi-local measure of congestion in networks a possible filtration parameter to guide network reconfiguration. We are currently developing an efficient way of detecting and classifying singularity formation under SRF using the techniques of persistent homology (PH). In this research we were guided by a quote:

"The theory of polyhedra and related geometrical methods are attractive not only in their own right. They pave the way for the general theory of surfaces. Surely, it is not always that we may infer a theorem for curved surfaces from a theorem about polyhedra by passage to the limit. However, the theorems about polyhedra always drive us to searching similar theorems about curved surfaces." A. D. Alexandrov 1950

\section{INTRODUCTION}

Combinatorial Ricci flow (CRF) on 2-dimensional surfaces has been used successfully over the past decade to solve many outstanding problems in fields spanning communications, medical imaging, computer vision and mathematics [1]. Any surface is conformally equivalent to one of three geometric surfaces depending on its genus; a sphere for genius 0 , a plane for genus one and a hyperbola for genus two and greater a consequence of the Uniformization Theorem (UT). CRF elicits this decomposition and thereby provides us with an efficiently-produced taxonomy for a surface. Armed with the success of CRF in two dimensions, and recognizing that the taxonomy is much finer in three dimensions (connected sum of prime manifolds from a set of eight homogeneous geometries); it was the objective of this research to generalize this approach and to develop an efficient and justifiable method to geometrize a given closed orientable 3-manifold. In particular we seek a unique and finite decomposition of the 3-manifold into a connected sum of prime geometries. Fortunately, Hamilton's Ricci flow (RF) was developed to elicit such a decomposition $[2,3,4,5,6]$. This was accomplished through a proof of Thurston's geometrization conjecture (GC) by Perelman in $2003[6,7]$. This can be done in a finite number of steps separated in time. In this sense, the GC is a 3-dimensional generalization of the UT. Thurston's GC states that each prime 3-manifold is either geometric or its simple pieces are geometric. However, this continuum mathematical approach is not numerically practical on such complex topologies and geometries. Therefore, we approached a solution to our objective by

rigorously developing a discrete PL version of Hamilton's Ricci flow for three and 
higher dimensions. We developed the first dimensionally agnostic generalization of $R F$ to piecewise linear simplicial spaces. We refer to our approach as simplicial Ricci flow (SRF) [8]. As part of this effort, we tested this approach on a representative spectrum of 3-geometries undergoing SRF $[9,10] .$. In particular, we studied numerically, (1) a 3-cylinder, (3) a 3-sphere at various resolutions, (3) Bianchi type IX geometry with one degree of anisotropy, and (4) the double lobed neck pinch geometry of Knopf et al. $[11,12]$ We demonstrated that each of our carefully-chosen examples numerically and reproduce the analogous continuum $\mathrm{RF}$ results $[9,10]$. In the pinched-neck 3-geometry, we specifically demonstrated that the SRF equations developed singularities for sufficiently pinched 3-geometries as in the continuum. We have developed an approach (SRF) that provides an efficient way of distinguishing 3-manifolds. Most recently, we showed analytically that the SRF equations converged to the continuum RF equations for the neck-pinch 3-geometry [10]. In this analysis we also showed that the error terms were explicitly second order and the error terms were bounded above (i.e. the detonators of the higher order terms were strictly positive).

A significant finding in our research was numerical stiffness of the SRF equations [9]. We understood the origins of this problem and circumvented it in our simulations by careful re-meshing of the 3-geometry. Nevertheless, this showed us the importance of having a sophisticated manifold sampling algorithm incorporating both adaptive meshing and re-meshing when needed. Our results are not unlike the difficulties encountered with CRF, and other finite element/volume codes. In these cases they need to keep the internal angles of their triangulation between careful bounds - the simplifies need to be fat in the sense of Withney's fullness criteria. In our simulations we were guided by two conditions when re-meshing, (1) we wanted the cells as fat as possible so that the circumcenter of each cell lies in the interior of its cell, and (2) we wanted all the deficit angles to be small ensuring that the cells would be large in lower curvature regions and smaller in higher curvature regions. These, sometimes competing conditions, require sophisticated manifold sampling techniques involving spline approximations to the tsimplicial surface. In the case of the neck-pinch geometry we utilized cubic splines to re-mesh. This was necessary in order to evolve the the neck pinch.

In order to efficiently identify singularity formation in SRF we are currently applying the methods of persistent homology to a selection of two and three-dimensional geometries evolved by simplicial Ricci flow [13]. To implement persistent homology, we constructed a triangular mesh for a sample of points. The scalar curvature along the edges of the triangulation, computed as an average of scalar curvatures at the endpoints of the edges, serves as a filtration parameter at each time step. We analyzed the results of the application of persistent homology to a two-dimensional rotational solid that collapses and three-dimensional dumbbells that manifest neckpinch singularities as presented in previous literature. We compared the appearance of critical geometric phenomena in these models with the results of the application of persistent homology and we concluded via various resolutions that persistent homology does indicate geometric criticality.

One of our graduate students (S. Ray) applied SRF to solve an outstanding problem in 
numerical analysis - the 1916 Weyl isometric embedding problem [14]. Our approach introduces a novel adiabatic isometric mapping (AIM) algorithm which is a numerical realization of Alexandrov's proof for embedding convex polyhedral metrics. In AIM the uniformization of the polyhedral surface under SRF induces a dimensional reduction of our problem that enables us to decouple our embedding equations equations on the spherical polyhedron - the endpoint of the SRF. We then use the reversibility of the SRF map to adiabatically pull back the coordinates from the spherical polyhedron to the original polyhedron. Our results are now being used by numerous groups to develop a discrete quasi-local measure of congestion in networks. This quasi local congestion (QLC) measure could be an ideal filtration parameter to guide network reconfiguration and ensure load balance.

\section{METHODS, ASSUMPTIONS AND PROCEDURES}

\subsection{Five Metrics and Milestones and Accomplishments.}

We addressed four primary research metrics throughout this research effort. First, (Metric 4.1-4.1.5 of proposal ) We had a kickoff meeting held in SUNY Stony Brook, and reported developments on the dimensionally agnostic simplicial version of the RF equations. The definition of these SRF equations required a subtile definition of the Ricci tensor as we needed to project them from the dual edges on the edges of the simplicial geometry. These equations were successfully benchmarked on a spectrum of models and shown to be consistent with the continuum Hamilton's RF equations. In particular we applied our equations to the 600-cell 3-sphere and to the Angenentknopf double-loaned axisymmetric neck pinch geometry as proposed. Extensions to non isotropic perturbations were also investigated successfullyy for the 600-cell model [15]. Secondly, (Metric 4.2.1-4.2.2) S-T Yau developed a generalization of curvature on graphs and applied this to an analysis of quantum tunneling on graphs $[16,17]$. The novel conclusion reached was that the single important factor in quantum diffusion on graphs was graph symmetry, and not locality. Thirdly, (Metric 4.3.1-4.3.3) we were forced to utilize diffeomorphism freedom in SRF in order to evolve our neck-pinch 3-geometry to a singularity. In particular, we needed to construct local cubic splines in order to re-mesh our geometry around the neck-pinch region in order to keep the circumcenters of the cells within the respective frustum block. Finally (Metric 44.1 and 4.4.2), we applied the SRF equations to efficiently solve an outstanding problem of the isometric embedding of surfaces into Euclidean 3-space. This application is being used to define a quasi-local definition of congestion in networks. This definition mirrors the Wang and Yau definition for quasi-local energy and momentum in general relativity. In addition, we have coupled the output of SRF to the input of the Perseus persistent homology code in oder to discriminate singularity formation in SRF. Our preliminary results show unique signatures for both Type- 1 and Type- 2 singularities in the neck-pinch model. If robust, this will substantially reduce the computational resources we need to meet our primary objective - the efficient and justifiable procedure for distinguishing 3-manifolds as a finite sums of connected prime manifolds.

Our fifth milestone (Metric 4.5.1-4.5.4) involve reporting our results. We highlight be- 
low the eleven publications related to this effort, including the seminal paper defining SRF in the 2014 issue of Comm. Math. Phys. Our research on SRF was presented at talks within the last month of this effort at the University of Texas at Austin and as a colloquium at the Mathematics Department at Florida Atlantic University. We held meetings at both SUNY Stony Brook, Harvard University and at the Griffiss Institute. Numerous seminars were given at Harvard, Rutgers University, FAU and Stony Brook. An invited talk was given at an International $\pi$ Mathematics conference at UBC in British Columbia.

We met or exceeded all five metrics and milestones described above. The community has acknowledged that we have developed the first faithful simplicial representation of Hamilton's Ricci Flow in arbitrary dimension. Exciting applications are underway both of pure mathematical foundation as well as applied complex network problems.

\subsection{Publications Under this Effort}

There are 10 publications in refereed journals associated to this effort, two poster contributions, and ten seminars. The publications are as follows:

1. Miller, W. A., McDonald, J. R., Alsing, P. M., Gu, X. D. and Yau, S-T, "Simplicial Ricci Flow," Commun. Math. Phys. 329 579-608 (2014).

2. McDonald, J. R., Miller, W. A., Alsing, P. M., Gu, X. D., Wang, X., and Yau, S-T., "On exterior calculus and curvature in piecewise-flat manifolds" Submitted to J. Math. Phys. (2012) arxiv.org/abs/1212.0919 [math.DG].

3. Alsing, P. M., Miller, W. A., Corne, M., Gu, X. D., Lloyd, S., Ray, S. and Yau, S-T, "Simplicial Ricci Flow: An Example of a Neck Pinch Singularity in 3D," Geom., Imaging Comp. in press (2014) ; arXiv:1308.4148 [math.DG].

4. Ray, S., Miller, W. A., Alsing, P. M. \& Yau, S-T, "Adiabatic Isometric Mapping Algorithm for Embedding Polyhedral Metrics in Euclidean 3 Space," to be submitted to Class. Quantum Grav. (2014).

5. Tichy, W., McDonald, J. R. and Miller, W. A., "New efficient algorithm for the isometric embedding of 2-surface metrics in 3 dimensional Euclidean space," Class. Quant. Grav. in press (2014).

6. Alsing. P., Blair, H. A., Corne, M., Jones, G., Miller, W. A., Mischaikow, K. and Nanda, V. "Topological Signatures of Singularities in Simplicial Ricci Flow," to be submitted to J. Comp. Geom. (2014).

7. Rui Shi, Mayank Goswami, Jie Gao, Xianfeng Gu Is Random Walk Truly Memoryless Traffic analysis and source location privacy under random walks, INFOCOM (2013).

8. Siming Li, Wei Zeng, Dengpan Zhou, Jie Gao Compact Conformal Map for Greedy Routing in Wireless Mobile Sensor Networks, INFOCOM (2013).

9. Xiaomeng Ban, Mayank Goswami, Wei Zeng, Xianfeng Gu, Jie Gao Topology Dependent Space Filling Curves for Sensor Networks and Applications, INFOCOM (2013). 
10. Wei Zeng, Huibin Li, Jean-Marie Morvan, Liming Chen, David Gu Xianfeng, An Automatic 3D Expression Recognition Framework based on Sparse Representation of Conformal Images, 10th IEEE International Conference on Automatic Face and Gesture Recognition, FG (2013).

11. Y. Lin, G. Lippner and S-T Yau, "Quantum Tunneling on Graphs," Commun. Math. Phys. 311, 113-132 (2012).

12. W. A. Miller, P. Alsing, M. A. Corne \& S. Ray, "Equivalence of Simplicial Ricci Flow and Hamilton's Ricci Flow for 3D Neckpinch Geometries," Geom., Imaging and Comp. (2014) in press.

\section{RESULTS AND DISCUSSION}

\subsection{Ricci Flow on Simplicial Geometries: the SRF Equations}

Here we highlight the important features of our construction of a discrete form of Hamilton's Ricci flow (RF) equations for a $d$-dimensional piecewise flat simplicial geometry, $\mathcal{S}$. These new algebraic equations are derived using the discrete formulation of Einstein's theory of general relativity known as Regge calculus (RC) [20]. A simplicial Ricci flow (SRF) equation can be associated to each edge, $\ell$, of a simplicial lattice. In defining this equation, we found it convenient to utilize both the simplicial lattice $\mathcal{S}$ and its circumcentric dual lattice, $\mathcal{S}^{*}$. As rotations occur in a polygon $h^{*} \in \mathcal{S}^{*}$. It is most natural to define the Ricci tensor along an edge $\lambda$ in the boundary of $h^{*}$ in this dual lattice. We refer to this as a "dual edge." In a $d$-dimensional simplicial lattice $\mathcal{S}$ the hinges are $(d-2)$-dimensional simplicies. The dual edge $\lambda$ is dual to a $(d-1)$-dimensional simplex. This simplex has $d+1$ hinges, $h$ in its boundary. The dual-edge Ricci tensor, $R c_{\lambda}$ is naturally defined as a certain weighted sum over these $d-1$ hinges $h$. We showed in [8] that the dual-edge SRF equation is naturally expressed as the proportionality between (1) the dual edge simplicial Ricci tensor, $R c_{\lambda}$, associated with the edge $\lambda \in \mathcal{S}^{*}$, and (2) a certain volume weighted average of the fractional rate of change of the edges, $\lambda \in \ell^{*}$, of the circumcentric dual lattice, $\mathcal{S}^{*}$. The inherent orthogonality between elements of $\mathcal{S}$ and their duals in $\mathcal{S}^{*}$ provide a simple geometric representation of Hamilton's RF equations. The foundation of our work under this grant is based on the following definition and associated corollary:

Definition 1. We define the dual-edge Regge-Ricci flow equation for any compact, piecewise-flat simplicial geometry, $\mathcal{S}$, as an equation for each edge, $\lambda$, in the circumcentric dual lattice, $\mathcal{S}^{*}$,

$$
\left(\begin{array}{c}
\text { Fractional rate of change } \\
\text { of a circumcentric } \\
\text { dual edge, } \\
\frac{1}{\lambda} \frac{\partial \lambda}{\partial t}
\end{array}\right)=-\left(\begin{array}{c}
\text { Ricci tensor associated } \\
\text { to the circumcentric } \\
\text { dual edge, } \\
R c_{\lambda}
\end{array}\right) \text {. }
$$

The dual edge SRF equation can then be projected onto an edge $\ell \in \mathcal{S}$ by taking a certain weighted sum over all the $(d-1)$-dimensional simplicies sharing edge $\ell$. 
Corollary 1. The simplicial Regge-Ricci flow equation for any compact, piecewiseflat simplicial geometry, $\mathcal{S}$, can be expressed as an equation for each edge, $\ell \in \mathcal{S}$,

$$
\left(\begin{array}{c}
\text { The volume averaged } \\
\text { fractional rate of change } \\
\text { of the dual edge, } \\
\left\langle\frac{1}{\lambda} \frac{\partial \lambda}{\partial t}\right\rangle_{\ell}
\end{array}\right)=-\left(\begin{array}{c}
\text { Ricci tensor associated to } \\
\text { the simplicial edge, } \\
R_{c_{\ell}}
\end{array}\right) \text {. }
$$

This was proved in [8].

We organize our results and discussion in this subsection into four sections. First, we express the Hamilton's RF equations in a mixed tetrad form that will be most convenient to apply to our simplicial geometry. Second, we define curvature on the simplicial lattice and construct the simplicial Ricci tensor. Third, we then construct the dual-edge $(\lambda)$ SRF equation and project this onto an edge $\ell$ in the simplical lattice $\mathcal{S}$ to prove the corollary. Finally, we solve these equations for a few illustrative examples and show that they reproduce the continuum Hamilton RF results to second order in the lattice. .

\subsection{Tetrad Formulation of Hamilton's Ricci Flow Equation}

The RF equation of a $d$-dimensional Riemannian geometry was first introduced by Hamilton [30],

$$
\frac{\partial g_{\mu \nu}}{\partial t}=-2 R_{\mu \nu}
$$

where $R_{\mu \nu}$ are the covariant components of the Ricci tensor $R c$, and $g_{\mu \nu}$ are the components of the metric tensor. It was our goal to reconstruct the RF equation of Hamilton on a piecewise-flat simplicial geometry, $\mathcal{S}$, by using the formalism of RC [20]. To this end, and without loss of generality, we consider a point $p \in\{\mathcal{M}, g\}$ where we can construct a time-independent orthogonal basis, $\left\{\boldsymbol{e}_{a}\right\}, \forall a=1,2 \ldots d$, i.e.

$$
\dot{\boldsymbol{e}}_{a} \equiv \frac{\partial \boldsymbol{e}_{a}}{\partial t}=0 .
$$

The contravariant components of the metric tensor in this basis,

$$
\boldsymbol{g}=\boldsymbol{e}_{a} g^{a b} \boldsymbol{e}_{b},
$$

will be, by construction, diagonal,

$$
g^{a b}=\operatorname{diag}\left\{g^{a a}\right\} .
$$

In this specially-chosen basis (Eq. 4) the time rate of change of the metric will be expressed solely in terms of the time derivatives of its $d$ diagonal components.

$$
\dot{\boldsymbol{g}}=\boldsymbol{e}_{a} \dot{g}^{a b} \boldsymbol{e}_{b}=\boldsymbol{e}_{a} \dot{g}^{a a} \boldsymbol{e}_{a}
$$

We found it convenient for our purposes to express the RF equation (Eq. 3) in its mixed-index form, i.e. as the components of a vector-valued 1-form equation in this 
basis at point $p$ in the sense of Cartan geometry [31]. The components of these mixedindex RF equations take a simple form,

$$
g^{a a} \dot{g}_{a a}=-2 R_{a}^{a} .
$$

Since the metric at point $p$ is diagonal then, $g^{a a}=1 / g_{a a}$, and the RF equation takes a simple form,

$$
\left(\begin{array}{c}
\text { Fractional rate of } \\
\text { change of } g_{a a}
\end{array}\right)=\frac{\dot{g}_{a a}}{g_{a a}}=-2 R_{a}^{a} .
$$

This mixed index tetrad equation forms the basis of the SRF equations.

\subsection{Curvature of a Piecewise-Flat $d$-Dimensional Simplicial Geometry: the $R m$ and $R c$ tensors}

In this section we provide a brief review of the curvature on a piecewise-flat simplicial lattice with emphasis on its application to the SRF equation. Simply put, we wish to define the dual SRF equation using Eq. 9 with $a=\lambda$. We motivate this in this section. The results rely critically on the mutual orthogonality of the simplicial lattice, $\mathcal{S}$ and its dual $\mathcal{S}^{*}$.

Although a more complete and thorough description will appear in the literature [8, 29], we felt that a review would make the derivation more complete for this final report. In particular, we briefly construct of the $R m$ and then the $R c$ on the simplicial lattice as well as its circumcentric dual lattice. We find it convenient to leverage the framework of RC [20, 32, 33, 34, 35, 36]. However, an essentially equivalent treatment of curvature on piecewise-flat simplicial manifolds is afforded by recent developments in DEC [22]. Both approaches, RC and DEC, appear to have independently encoded the geometry and curvature structure on discrete simplicial lattices in similar ways, and both utilize the circumcentric dual lattice in a fundamentally important way $[8,29]$.

In the Euclidean form of RC the geometry of the interior of each of its $d$-dimensional simplicies is assumed to be flat Euclidean space, $E^{d}$. The geometry of each simplex in $\mathcal{S}$ is completely determined by the square of its $\left(\begin{array}{l}d \\ 2\end{array}\right)=\frac{d(d-1)}{2}$ edge lengths. Therefore, the entire lattice geometry and its curvature structure is completely determined by the squared length of all its edges, $\left\{\ell_{i}^{2}\right\}$, and only these edges. The set of all edge lengths of the simplicial geometry is the simplicial analogue of the metric, i.e.

$$
\boldsymbol{g} \longleftrightarrow\left\{\ell_{i}^{2}\right\}
$$

Any geometric quantity on the lattice, e.g. its lengths, areas, volumes, curvatures, and even the dual lattice are defined solely in terms of these edge lengths and nothing else.

The circumcentric dual lattice is fundamental to RC [32, 33, 38, 39] and DEC. Consider a $d$-dimensional simplicial lattice, $\mathcal{S}$. Each vertex forming $\mathcal{S}^{*}$ lies at the center of a $d$-simplex in $\mathcal{S}$. The dual edges $\lambda \in \mathcal{S}^{*}$ connect adjacent circumcentric vertices across a common $(d-1)$-simplex. This edge is perpendicular to this $(d-1)$ simplex face. Consider a compact simplicial lattice $\mathcal{S}$ and its circumcentric dual $\mathcal{S}^{*}$. For simplicity, and not out of necessity, we consider only compact $d$-dimensional simplicial 
geometries in this final report. This could be extended to geometries with boundaries by utilizing previous results in RC [40]. Furthermore, we also assume here, for the same reasons, that $\mathcal{S}$ is a well-centered Delaunay lattice [37] and $\mathcal{S}^{*}$ is its Voronoi lattice. The vertices, edges, triangles, ... and $d$-simplexes in $\mathcal{S}$ will be denoted by $\sigma_{0}, \sigma_{1}, \sigma_{2}, \ldots, \sigma_{d}$; respectively. The geometry of each simplex is determined uniquely by its $\left(\begin{array}{l}d \\ 2\end{array}\right)$ squared lengths; consequently the geometry of the entire simplicial lattice, $\left\{\ell_{i}^{2}\right\} \in \mathcal{S}$ is a function of all the edge lengths. Similarly, we label the vertices, edges, polyhedra and polytopes of $\mathcal{S}^{*}$ by $\sigma_{d}^{*}, \sigma_{d-1}^{*}, \sigma_{d-2}^{*}, \ldots, \sigma_{1}^{*}, \sigma_{0}^{*}$, respectively. The geometry of each of these dual cells owes its existence, and can be expressed as, functions of the squared edge lengths of $\mathcal{S}$. The subscript on the simplicial elements is its dimension, e.g. $\sigma_{k} \in \mathcal{S}$ is a $k$-simplex; where the subscript on the circumcentric dual polytope is its co-dimension, e.g. $\sigma_{k}^{*} \in \mathcal{S}^{*}$ is the $(d-k)$-dimensional circumcentric dual element of $\sigma_{k} \in \mathcal{S}$. Perhaps the most important property of the Voronoi and Delaunay lattices is that each element $\sigma_{k} \in \mathcal{S}$ is perpendicular to its dual element $\sigma_{k}^{*} \in \mathcal{S}^{*}$.

We have discovered that the $d$-volume formed by connecting the vertices of the $k$ simplex, $\sigma_{k}$, to the vertices of the $(d-k)$-dimensional polytope forms what we refer to as a $d$-dimensional hybrid polytope, $V_{k k^{*}}$, of volume,

$$
\left|V_{k k^{*}}\right|=\left\langle\sigma_{k} \mid \sigma_{k}\right\rangle=\int \sigma_{k} \wedge \sigma_{k}^{*}=\left(\begin{array}{l}
d \\
k
\end{array}\right)^{-1}\left|\sigma_{k}\right|\left|\sigma_{k}^{*}\right|=\frac{k !(d-k) !}{d !}\left|\sigma_{k}\right|\left|\sigma_{k}^{*}\right|,
$$

and is essential to constructing curvature tensors on the simplicial lattice. That this $d-$ volume is a simple product of the volume of the dual-cell pairs reflects an important property of RC and is due to the orthogonality between the simplicial and circumcentric dual lattice. In addition, these $d$-hybrid volumes, when applied to all pairs of $k$-dual cells $\left\{\sigma_{k}^{*}, \sigma_{k}\right\}$, provide a proper tiling of the discrete geometry. The curvature of the simplicial lattice has compact support on such hybrid volumes, e.g. the $R m$ is naturally defined on $V_{h h^{*}}$, the $R c$ on $V_{\lambda \lambda^{*}}$, and the scalar curvature, $R$, on $V_{v v^{*}}$, where $h$ is the $(d-2)$-dimensional hinge in $\mathcal{S}, \lambda$ is a dual edge in $\mathcal{S}^{*}$, and $v$ is a vertex in either $\mathcal{S}$ or $\mathcal{S}^{*}[22,34,35,36]$. We provide an illustration of the five hybrid volumes in the case of a 4-dimensional lattice in Fig. 1.

In Fig. 1 each of the five rows in this table illustrates one of the five hybrid 4dimensional polytopes. The black edges (thick lines) in the diagram are in the simplicial Delaunay lattice, $\mathcal{S}$ while the red (thick grey) edges are in the circumcentric dual Voronoi lattice, $\mathcal{S}^{*}$. The blue (thin) edges are added in the right-most column and connect the vertices of the Delaunay lattice to the vertices of the Voronoi lattice thus forming a 4-dimensional polytope. Each of these 5 polytopes, when applied throughout all similar elements of $\mathcal{S}$, provide a complete tessellation of the lattice spacetime. The symbols $V_{k k^{*}}$ in the left column completely characterize the hybrid polytope in the $d$-dimensional lattice. The first number, $k$, is the $k$-dimensional simplicial element $\sigma_{k} \in \mathcal{S}$, while the second starred number is its co-dimension $k$ dual polytope in $\sigma_{k}^{*} \in \mathcal{S}^{*}$. In the center column, we show the $k$-dimensional simplicial element in the black line, and its dual $(d-k)$-dimensional element in $\mathcal{S}^{*}$ is shown in red (grey). 


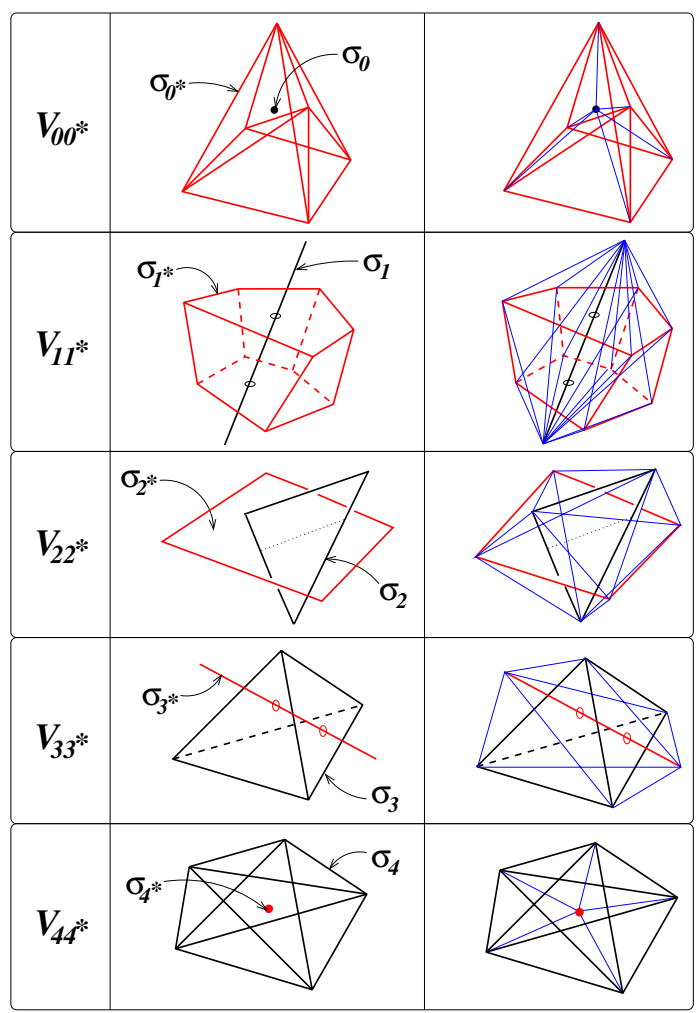

Figure 1: The five hybrid 4-dimensional polytopes.

On a simplicial lattice, it is well known that the curvature is concentrated on the (d2 )-dimensional hinges $h$ [20]. The form of this curvature is a conic singularity at the hinge. One approach used by researchers is to rigorously analyze this singularity structure, explicitly constructing a Dirac delta distribution for the curvature on the hinge $h$ by embedding the hybrid block and its simplicial support into a $(d+1)$ dimensional Euclidean space [32, 41]. This Dirac delta distribution is assumed to be uniformly distributed over the hinge, $h$, and thus integral curvature measures were derived. An alternative and equivalent description has recently been used $[8,29,33$, $34,35,36,39]$. First, we take the support for this curvature to be uniformly distributed over the entire hybrid block. Second, we use the Voronoi area, $h^{*}$ and only this area to define the support for the sectional curvature. Finally, we assume the geometry within each hybrid block, $V_{h h^{*}}$, is an Einstein space [42]. These approaches yield curvature components within each hybrid cell that are proportional to each other, and can be transformed consistently to each other by taking suitable traces or identifying certain eigenvalues. It is important to emphasize that these are hybrid curvatures, and not the curvature tensors that are found in the RF and SRF equations. They refer to one of the sectional curvatures and not a trace that is required to define the Ricci tensor.

In the remainder of this final report we assume that the components of curvature within the $V_{(d-2)(d-2)^{*}}$ hybrid block can be treated as an Einstein space, and we define the Gaussian curvature by the parallel transport of vectors restricted to the perimeters of the dual Voronoi polygons, $h^{*}=\sigma_{d-2}^{*}$ where $h^{*}$ is the dual polygon to the $(d-2)-$ 
dimensional simplicial hinge, $h$. In this sense, the area of the dual Voronoi polygon, $h^{*}$, becomes the unique weighting area associated to hinge $h$ in order to define the Gaussian curvature,

$$
\left(\begin{array}{c}
\text { Gaussian curvature } \\
\text { associated to hinge } h
\end{array}\right)=K_{h}=\frac{\text { Angle Vector Rotates }}{\text { Area Circumnavigated }}:=\frac{\epsilon_{h}}{h^{*}},
$$

where $\epsilon_{h}$ is the deficit angle associated to hinge $h$, and $h^{*}$ is the area of the Voronoi plane dual to hinge $h$. This $V_{(d-2)(d-2)^{*}}$ hybrid cell is defined as the set of points in the lattice closer to the hinge $h$ than to any other hinge and provides a reasonable support for this curvature. In the next three subsections we briefly reexamine the construction of the Riemann $(R m)$, Ricci $(R c)$ and scalar curvature $(R)$ on a simplicial lattice beginning as a guide with their respective definitions from the continuum.

For notational purposes, whenever we write a volume using a capital $V$, it will be understood that this represents a $d$-dimensional volume. It can represent a $d$-dimensional hybrid or reduced-hybrid volume. Its subscript will identify to which volume it corresponds. For example, the hybrid cell $V_{(d-2)(d-2)^{*}}$ associated to hinge $h$ will be written as $V_{h}=V_{(d-2)(d-2)^{*}}$. On the other hand, if we write, simply, $h$, in the correct context with no capitol, $V$, then it will mean the volume of $h=|h|$. Our notation follows similarly for all other elements, e.g. $\ell=|\ell|$ for the length of an edge, $h^{*}=\left|h^{*}\right|$ for the area of the Voronoi polygon as in Eq. 12.

Now we utilize the local Einstein-space structure within each hybrid cell, and construct the $R m$ on $\mathcal{S}$ is by examining the parallel transport of a vector around a closed loop. In particular, a vector, $\boldsymbol{v}:=v^{a} \boldsymbol{e}_{b}$ will ordinarily return rotated by an amount, $\boldsymbol{e}_{a} \delta v^{a}$, when parallel transported around the boundary of the area element, $A^{c d} \boldsymbol{e}_{c} \wedge \boldsymbol{e}_{d}$,

$$
\delta v^{a}=-2 R_{b c d}^{a} v^{b} A^{c d} .
$$

This defines a constructive way to explore the components of the $R m$ through the rotation operator.

$$
\mathcal{R}=\frac{1}{4} \underbrace{\boldsymbol{e}_{a} \wedge \boldsymbol{e}_{b}}_{\begin{array}{c}
\text { rotation } \\
\text { bivector }
\end{array}} \underbrace{R^{a b} c d}_{R m} \underbrace{\boldsymbol{R}^{c} \wedge \boldsymbol{e}^{d}}_{\begin{array}{c}
\text { loop of } \\
\text { circum- } \\
\text { navigation }
\end{array}} .
$$

The curvature of a $d$-dimensional piecewise flat simplicial manifold is concentrated on its co-dimensional 2 simplicial hinges, $h$ [20]. Given any hinge, $h=\sigma_{d-2} \in \mathcal{S}$, there will be at least three $d$-simplicies sharing hinge $h$. We assume here that there are $n \geq 3$ of these hinging on $h$, and we label these simplicies $\left\{\sigma_{d}(i)\right\}$ for $i=1,2, \ldots, n$. Let us consider a loop, $\mathcal{C}$, within the interior of the collection of these $n d$-simplicies that encircles $h$ only once. In addition, we consider a vector $\boldsymbol{v}$ at a point $p_{0}$ along $\mathcal{C}$. We place $p_{0}$ at the intersection of $\mathcal{C}$ and the $(d-1)$-simplex , $\sigma_{d-1}(1,2)$ that is shared by the two adjacent $d$-simplicies, $\sigma_{d}(1)$ and $\sigma_{d}(2)$. The parallel transport of this vector within $\sigma_{d}(1)$ is trivial since the geometry is flat. The angle $v$ makes with the $d(d+1) / 2$ 
edges of $\sigma_{d}$ remains constant as it is parallel transported along $\mathcal{C}$. Furthermore, as we continue to parallel transport the vector along $\mathcal{C}$ into $\sigma_{d}(2)$ it is equally trivial. There is no curvature concentrated at their juncture $\left(\sigma_{d-1}(1,2)\right)$, i.e. the two adjacent $d$ simplicies can be embedded into the same flat Euclidean geometry. We can continue to parallel transport $\boldsymbol{v}$ along $\mathcal{C}$ through the remaining $(n-2) d$-simplicies trivially; however, when we return to the starting point $p_{0}$ on $\mathcal{C}$ we will find the vector rotated by the deficit angle $\epsilon_{h}$,

$$
\epsilon_{h}:=2 \pi-\sum_{i=1}^{n} \theta_{i}
$$

where the summation is over all $\sigma_{d}(i)$ sharing hinge $h$, and $\theta_{i}$ is the hyperdihedral angle between the two $(d-1)$-dimensional simplicial faces $\sigma_{d-1}(i-1, i)$ and $\sigma_{d-1}(i, i+1)$ of simplex $\sigma_{d}(i)$ sharing hinge $h$. Remarkably, this rotation bivector lies in the plane, $h^{*}=\sigma_{2}^{*}$ perpendicular to hinge $h$, and this rotation is independent of the orientation and area of $\mathcal{C}$. This captures the integrated curvature at hinge $h$. The curvature is a conic singularity. The closed curve $\mathcal{C}$ can be shrunk to an arbitrarily small area, $A_{\mathcal{C}}$, around $h$ without affecting the rotation in $v$ under parallel transport. Therefore the sectional curvature can be made as large as one pleases by shrinking the area,

$$
\left(\begin{array}{c}
\text { Conical Sectional } \\
\text { Curvature of hinge } \\
h \in \mathcal{S}
\end{array}\right)=\left(\begin{array}{c}
\text { Angle vector rotates } \\
\text { Area circumnavigated }
\end{array}\right)=\lim _{A_{\mathcal{C}} \rightarrow 0} \frac{\epsilon_{h}}{A_{\mathcal{C}}}=\infty .
$$

Is there a natural area that we can assign to the hinge, $h$ ? What area does RC use? To answer these questions, we identify three features of $\mathcal{S}$ that yield a unique curvature measure. First, the simplicial geometry is piecewise flat. Second, each element of simplicial geometry, $\sigma_{k} \in \mathcal{S}$ is orthogonal to its circumcentric dual element, $\sigma_{k}^{*} \in$ $\mathcal{S}^{*}$. Thirdly, it has been shown that the Regge equations as well as the Regge action are uniquely defined as distributed quantities over such circumcentric dual elements $[33,34,43]$. In his original paper on RC, Regge demonstrated that the curvature of a simplicial lattice is concentrated at each of the co-dimension 2 hinges, $h=\sigma_{d-2} \in \mathcal{S}$, and this curvature at each $h$ is a conic singularity as illustrated by Eq. 16. However, in $\mathrm{RC}$ it appears that this curvature is defined to be distributed uniformly over the convex hull of the triangle hinge $h$ and the polygon, $h^{*}=\sigma_{2}^{*} \in \mathcal{S}^{*}$ dual to $h$. Following the notation introduced in the end of last section, this area, $h^{*} \in \mathcal{S}^{*}$ is central to the hybrid cell $V_{h}:=V_{22^{*}}$, and this hybrid cell is the set of points in $\mathcal{S}$ closest to hinge $h$ than to any other hinge. The area circumnavigated is, $h^{*}:=\left|h^{*}\right|$ since $\mathrm{RC}$ defines the loop $\mathcal{C}$ to be the perimeter of the dual polygon $h^{*}$. This area, $h^{*}$ and the deficit angle, $\epsilon_{h}$, are both functions of the squared edges, $\left\{\ell_{i}^{2}\right\}$ of $\mathcal{S}$. This (1) dual area $h^{*} \equiv\left|h^{*}\right|$, and (2) rotation, $\epsilon_{h}$, yield the hinge-based (or equivalently dual hinge-based) sectional curvature in $\mathrm{RC}$,

$$
K_{h}=K_{h^{*}}=\frac{\epsilon_{h}}{h^{*}}
$$

In order to use the continuum equations, Eqs. 13-14 we need to construct an appropriate set of basis vectors within the $V_{h}$ hybrid cell defined by the convex hull of $h$ and $h^{*}$. 
Following the development in Sec. 4.2, we will find it convenient to define a time independent $d$-dimensional frame of orthogonal bases 1-forms, $\left\{\boldsymbol{e}^{a}\right\}$ for $a=1,2, \ldots, d$, and corresponding basis vectors, $\left\{\boldsymbol{e}_{a}\right\}$ for $a=1,2, \ldots, d$, that span a given hybrid polytope $V_{h h^{*}}$,

$$
\boldsymbol{e}_{a}=g_{a b} \boldsymbol{e}^{b} .
$$

We refer to the following basis as the $\ell \lambda$-hybrid basis. We first identify an edge $\ell \in h$ and a dual edge $\lambda \in h^{*}$ in $V_{h}$. If we connect the vertices of this $\lambda$ to the $d$ vertices of the hinge $h$, this will form a reduced hybrid $d$-simplex that we will refer to as $V_{h \lambda}$, i.e. the hybrid cell $V_{h}$ restricted to $\lambda$. The sum of all $V_{h \lambda}$ 's for each $\lambda \in h^{*}$ equals the volume of the non-simplicial hybrid cell,

$$
V_{h}=\sum_{\lambda \in h^{*}} V_{h \lambda} .
$$

We define our orthogonal basis on each of these reduced hybrid cells, $V_{h \lambda}$. In order to capture the orthogonality between $\mathcal{S}$ and $\mathcal{S}^{*}$ within the hybrid cell $V_{h}$, we define basis vectors of four kinds.

$$
\boldsymbol{e}_{a}=\left\{\begin{array}{lll}
\boldsymbol{e}_{\ell}=\vec{\ell}, & & \ell \in h, \\
\boldsymbol{e}_{i}=\overrightarrow{m_{i}}=\overrightarrow{\mathcal{O}_{i} \mathcal{O}_{i+1}}, & & \forall i \in\{1,2, \ldots, d-3\}, \\
\boldsymbol{e}_{\lambda}=\vec{\lambda}, & & \lambda \in h^{*}, \\
\boldsymbol{e}_{h \lambda}=\overrightarrow{m_{h \lambda}}, & &
\end{array}\right.
$$

where,

- $\mathcal{O}_{1}$ is the circumcenter of edge $\ell \in h$,

- $\mathcal{O}_{2}$ is the circumcenter of any one of the triangles, $\sigma_{2}$, in $h$ such that $\sigma_{2} \ni \ell$,

- $\mathcal{O}_{3}$ is the circumcenter of any one of the tetrahedra in $h$ such that $\sigma_{3} \ni \sigma_{2}$,

- $\mathcal{O}_{d-3}$ is the circumcenter of one of two the (d-3)-dimensional simplexes in $h$ such that $\sigma_{d-3} \ni \sigma_{d-4}$,

- $\mathcal{O}_{d-2}$ is the circumcenter of the $(d-2)$-dimensional simplicial hinge, $h=\sigma_{d-2}$,

and, $m_{\ell \lambda}$ is the segment from $\mathcal{O}_{1}$ to the point on lambda, $\mathcal{O}_{\lambda}^{*}$, so that this line segment is perpendicular to $\lambda$, i.e. it intersects $\lambda$ at the circumcenter of the (d-1)-dimensional simplex, $\lambda^{*}$. The set of (d-2) mutually orthogonal vectors, ${ }^{1}$

$$
\left\{\vec{\ell}, \vec{m}_{i}\right\},
$$

\footnotetext{
${ }^{1}$ In 2-dimensions there the hinge is a vertex and the space is a panned by only two orthogonal vectors, $\left\{\vec{\lambda}, \vec{m}_{h \lambda}\right\}$, i.e. there are no $\vec{m}_{i}$ 's. In three dimensions there are also no $m_{i}$ 's and the space is a panned by the three orthogonal vectors, $\left\{\vec{\ell}, \vec{\lambda}, \vec{m}_{h \lambda}\right\}$. It is only in four and higher dimensions that we start generating the $m_{i}$ 's.
} 
span the hinge, $h=\sigma_{d-2}$, while the remaining two vectors,

$$
\left\{\vec{\lambda}, \overrightarrow{m_{h \lambda}}\right\}
$$

span the dual polyhedron, $h^{*}$. The hinge can be expressed as a (d-2)-vector,

$$
h=\frac{1}{(d-2) !}\left(\boldsymbol{e}_{\ell} \wedge \boldsymbol{e}_{1} \wedge \boldsymbol{e}_{2} \wedge \ldots \wedge \boldsymbol{e}_{d-3}\right),
$$

where the ( $d$-2)-volume of the hinge is $h=|\boldsymbol{h}|$. In addition, the dual hinge can be equivalently expressed either as the dual of Eq. 23 or by using the last two basis vectors in Eq. 22,

$$
h^{*}=h^{*} \frac{e_{\lambda} \wedge e_{h \lambda}}{\left|e_{\lambda} \wedge e_{h \lambda}\right|}=h^{*} \hat{h}^{*} .
$$

The contravariant components of this flat-space metric, $g$, in this vector basis is,

$$
g^{a b}=\operatorname{diag}(\underbrace{\ell^{-2}, m_{0}^{-2}, m_{1}^{-2}, \ldots, m_{d-3}^{-2}}_{\text {hinge }, h}, \underbrace{\lambda^{-2}, m_{h \lambda}^{-2}}_{h^{*}}) .
$$

A dual set of orthogonal basis 1-forms can be generated,

$$
\boldsymbol{\omega}^{a}=g^{a b} \boldsymbol{e}_{b}
$$

and the covariant components of the metric are just,

$$
g_{a b}=\operatorname{diag}(\underbrace{\ell^{2}, m_{0}^{2}, m_{1}^{2}, \ldots, m_{d-3}^{2}}_{\text {hinge }, h}, \underbrace{\lambda^{2}, m_{h \lambda}^{2}}_{h^{*}}) .
$$

We are now in a position to construct the $R m_{h}$ associated to hinge $h$ by examining the rotation operator, $\mathcal{R}$, defined by Eq. 14 . The sectional curvature, $K_{h}$, is naturally defined on the convex hull of $h$ and $h^{*}$, where the convex hull is just the hybrid volume, $V_{h}=V_{22^{*}}$ (as illustrated in Fig. 1 for the special case of 4-dimensions). Furthermore, this is the only nonzero sectional curvature within the hybrid polytope $V_{h}$. It is the orthogonality between any element of the simplicial lattice, $\sigma_{k}$, and its corresponding circumcentric dual, $\sigma_{k}^{*}$, that yields an Einstein space geometry within each hybrid cell, and that each of the $d(d-1)$ non-zero components of the $R m_{h}$ is proportional to the sectional curvature $K_{h}$ [34]. No matter what the orientation of the loop, $\Sigma$, we take encircling the hinge $h$, the unit rotation bivector will always lie in the plane of $h^{*}$ and have magnitude $\epsilon_{h}$,

$$
\text { ( . ) } \mathcal{R}(\Sigma)=\hat{h}^{*} \epsilon_{h} \text {. }
$$

Therefore, the only non-vanishing components of the simplicial $R m$ associated to hinge $h$ are when the first two indices lie in the dual polygon, $h^{*}$. While, it is true that 
in the basis chosen above the only non vanishing components of the $R m$ are in the Voronoi plane, $h^{*}$,

$$
R_{\lambda m_{\lambda}}^{\lambda m_{\lambda}}=\frac{\epsilon_{h}}{h^{*}} .
$$

Nevertheless, this would not describe adequately the curvature within the hybrid cell. In particular, any basis chosen in general position would ordinarily (except for a set of measure zero) not have a single vector that lies in the hyperplane spanned by $h$. This would yield a fully-populated rotation matrix. In particular, this would be true for our specially constructed basis if we rotated each of the basis vectors $\left\{\vec{\ell}, \vec{m}_{1}, \vec{m}_{2}, \ldots \vec{m}_{d}-3\right\}$ even slightly out of the plane of $h$. In this sense, the space within each hybrid cell, $V_{h}$, is a Einstein space [42]. Additionally, if we restrict the parallel transport of vectors around the perimeter of the Voronoi polygon, $h^{*}$, then we have usual Einstein space expressions for those components of the Riemann, $R m_{h}$, the Ricci, $R c_{h}$ and scalar $R_{h}$ curvatures associated exclusively to the Einstein space of each hybrid cell $V_{h}$,

$$
\begin{aligned}
R m_{h}^{(h y b)} & =K_{h}=\frac{\epsilon_{h}}{h^{*}}, \\
R c_{h}^{(h y b)} & =(d-1) K_{h}=(d-1) \frac{\epsilon_{h}}{h^{*}}, \text { and } \\
R_{h}^{(h y b)} & =d(d-1) K_{h}=d(d-1) \frac{\epsilon_{h}}{h^{*}} .
\end{aligned}
$$

Each of these Einstein space curvatures (Eqs. 30-32) associated with their hybrid cell are uniformly distributed within this cell. This interpretation is consistent with other derivations $[32,41]$ with proper care in their normalization. The component of the Riemann associated to the hinge-based hybrid cell is the entire Riemann,

$$
R m_{h}^{(h y b)}=R m_{h}
$$

as it realizes the entire sectional curvature attributable to the hinge $h$. Therefore, the Riemann tensor in Cartan language is,

$$
\boldsymbol{R} \boldsymbol{m}_{h}=\hat{\boldsymbol{h}}^{*} R m_{h} \hat{\boldsymbol{h}}^{*}=\hat{\boldsymbol{h}}^{*} K_{h} \hat{\boldsymbol{h}}^{*}=\hat{\boldsymbol{h}}^{*} \frac{\epsilon_{h}}{h^{*}} \hat{\boldsymbol{h}}^{*}
$$

where, $\hat{\boldsymbol{h}}^{*}=\boldsymbol{h}^{*} / h^{*}$ is the unit 2-form generated on $h^{*}$ in hybrid volume, $V_{h}$. In the $\ell \lambda$-hybrid basis this unit bivector is given by Eq. 24 .

We constructed the Riemann tensor and in the remainder of this section we examine the simplicial $R c$ on $\mathcal{S}$. This will involve the simplicial version of taking the trace of the simplicial $R m$ tensor in Eq. 30. We examine the $R c$ tensor here as it appears on the right hand side of the RF equation (Eq. 3). This tensor was first introduced in [32], and later in context within the hybrid cells by [36]. We briefly reconstruct this tensor by utilizing the Einstein space geometry interior to $V_{h}$, and by using the $\ell \lambda$-hybrid basis construction (Eq. 20) discussed in the previous section. This construction provides us with a geometric description of the trace operation on a lattice geometry. 


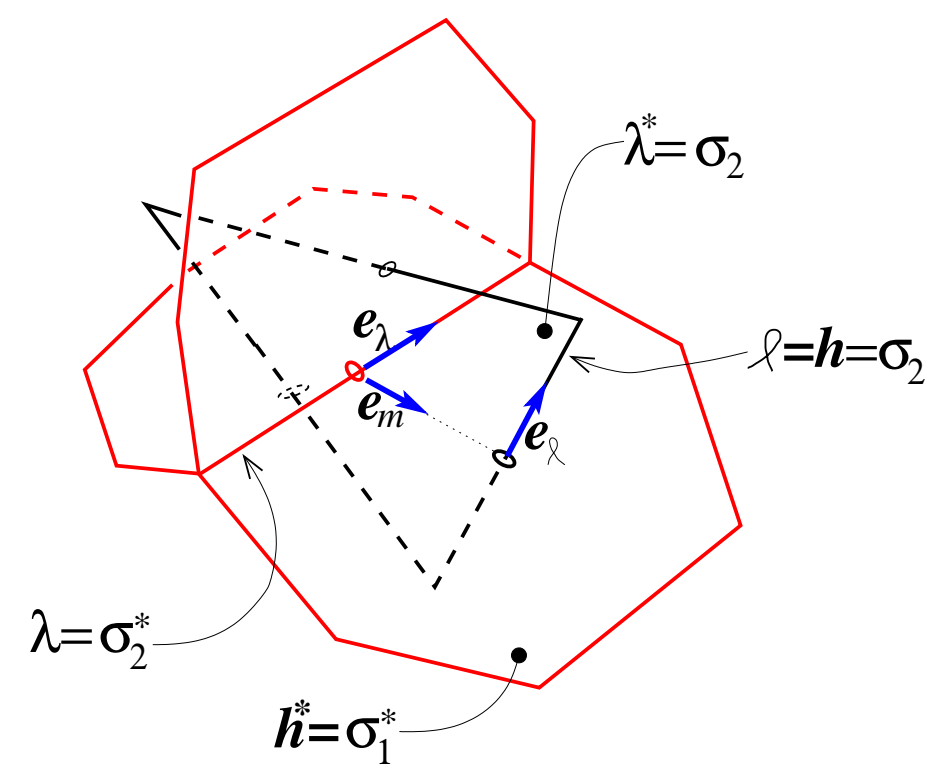

Figure 2: We illustrate here the lattice geometry used to define the $R c$ tensor for a 3-dimensional simplicial lattice.

In the continuum, the simplicial $R c$ is a 1 -form valued 1 -form,

$$
R c=e^{a} R_{a b} e^{b}=e^{a} R_{a c b}^{c} e^{b} .
$$

This tensor is defined along some basis vector, $\boldsymbol{e}_{b}$ in the tangent space. This basis vector defines a common set of area bivectors for circumnavigation, i.e. $\boldsymbol{\Sigma}=\sigma^{c b} \boldsymbol{e}_{c} \wedge \boldsymbol{e}_{b}$, one for each, $c \neq b$. In the previous section we defined the $R m_{h}$ in RC on the dual Voronoi areas, $h^{*}=\sigma_{d-2}^{*} \in \mathcal{S}^{*}$ of the hybrid cell, $V_{h}$. Each edge, $\lambda=\sigma_{d-1}^{*} \in \mathcal{S}^{*}$, is a common bounding edge of $d(d-1) / 2$ of these dual polygons, $h^{*}$. It is therefore natural to define a simplicial Ricci tensor, $R c_{\lambda}$, along these dual edges, $\lambda$. In this sense the trace of the $R m$ is a suitable sum over each of these Voronoi ares, $h^{*}$ sharing edge $\lambda$. We illustrate this in Fig. 2 for the case of three dimensions.

In Fig. 2 we illustrate the lattice geometry used to define the $R c$ tensor for a 3dimensional simplicial lattice. The $R c$ tensor is naturally defined on an edge, $\lambda=$ $\sigma_{1}^{*} \in \mathcal{S}^{*}$. Along this edge we define one of the unit triad vectors, $\hat{e}_{\lambda}$ of the $\ell \lambda$-basis, where $e_{\lambda}=\lambda \hat{e}_{\lambda}$ as usual. In this illustration, we show the three dual polygons in red, $\ell_{\mid \lambda}^{*} \in \mathcal{S}^{*}$ sharing edge $\lambda$. Dual to each of these polygons is an edge of the triangle $h=\lambda^{*} \in \mathcal{S}$. Along one of these edges, $\ell \subset h$, we define the second of the triad vectors, $\boldsymbol{e}_{\ell}=\ell \hat{\boldsymbol{e}}_{\ell}$. Finally the normalized vector from the center of edge $\ell$ perpendicular to edge $\lambda$, defines our final triad vector, $\boldsymbol{e}_{\ell \lambda}=m_{\ell \lambda} \hat{\boldsymbol{e}}_{m}$.

We construct the Ricci tensor, $R c_{\lambda}$, associated to a dual edge $\lambda$ by properly tracing over each of the Ricci tensors, $R c_{h}^{(h y b)}$, defined in association with each Voronoi polygon, $h^{*}$, containing dual edge $\lambda$. The trace, or sum, will be over all $h^{*}$ restricted to $\lambda$, or $h^{*}{ }_{\mid \lambda}$. The trace is accomplished using a weighted average as described for RC in $[34,35,36]$ and independently in discrete exterior calculus by [22]. Here one considers 
the component of the Ricci tensor associated to the hybrid cell, $R c_{\lambda}$, to be uniformly distributed over the hybrid $d$-volume of the simplicial lattice associated with edge $V_{\lambda}$ and integrates this over this volume. This integrated tensor can be identified with the trace of the familiar hinge-based expression for simplicial Riemann tensor, $R m_{h}$ in Eq. 31 with the inclusion of a proper weighting factor. In particular,

$$
R c_{\lambda} V_{\lambda}=\underbrace{\sum_{h_{\mid \lambda^{*}}} R c_{h}^{(h y b)} V_{\lambda h}}_{\begin{array}{c}
\text { simplicial form } \\
\text { of the trace }
\end{array}},
$$

where,

$$
\begin{aligned}
V_{\lambda} & =\left(\begin{array}{l}
d \\
1
\end{array}\right)^{-1} \lambda \lambda^{*}=\sum_{h_{\mid \lambda^{*}}}\left(\begin{array}{l}
d \\
2
\end{array}\right)^{-1} h h_{\lambda}^{*}, \\
V_{\lambda h} & =\left(\begin{array}{l}
d \\
2
\end{array}\right)^{-1} h h_{\lambda}^{*}, \quad \text { and } \\
R c_{h}^{(h y b)} & =(d-1) R m_{h}=(d-1) \frac{\epsilon_{h}}{h^{*}} .
\end{aligned}
$$

Hence,

$$
R c_{\lambda}=\frac{\sum_{h_{\lambda^{*}}} R c_{h}^{(h y b)} h h_{\lambda}^{*}}{\sum_{h_{\lambda^{*}}} h h_{\lambda}^{*}}:=\left\langle R c_{h}^{(h y b)}\right\rangle_{\lambda} .
$$

In constructing $R c_{\lambda}$ we make use of reduced hybrid blocks, $V_{h^{*} \lambda}$, as well as reduced hinge volumes, $h_{\lambda}^{*}$ which are illustrated in the case of $d=4$ in Fig. 3. In general, the reduced hybrid $d$-volume, $V_{h^{*} \lambda}$, is the fraction of the set of points interior to the hybrid $d$-volume, $V_{h^{*}}$ that are closest to the dual edge, $\lambda$. This provides a decomposition of the hybrid cell into reduced hybrid cells,

$$
V_{h}=\sum_{\lambda \in h^{*}} V_{h \lambda}
$$

as illustrated in Fig. 2. Each reduced hybrid cell, $V_{h \lambda}$, contain the circumcenters, $\mathcal{O}_{i}$, that defined the $\ell \lambda$-hybrid basis. Similarly, the reduced hinge is a ( $d-2)$-volume, $\lambda_{h}^{*}$, and is defined to be the set of points in the interior of the $(d-1)$-dimensional simplex, $\sigma_{\lambda}^{*}$, that are closest to its bounding hinge, $h$, than to any other hinge in its boundary.

In Fig. 3 we illustrate the various geometric elements that were used to define the $R c_{\lambda}$ in 4-dimensions. The line segment $\lambda=\overline{U V}$ is the edge in the circumcentric dual lattice, $\mathcal{S}^{*}$, where we define the Ricci tensor. The tetrahedron $\lambda^{*}=\{A B C D\} \in \mathcal{S}$ is perpendicular to $\lambda$. If we connect the endpoints of $\lambda$ to the four vertices of $\lambda^{*}$ the resulting 4-dimensional cell is the $\lambda$-hybrid simplex of volume, $V_{\lambda}=(1 / d) \lambda \lambda^{*}$. In 4-dimensions the hinges are triangles. We highlight one of the 4 triangles, $h=$ $\{A, B, C\} \subset \partial \lambda^{*}$, bounding $\lambda^{*}$ by shading the triangle. The point $O$ lies at the circumcenter of $\lambda^{*}$. It is the point equidistant from vertices, $A, B, C$ and $D$. Point $O$ 


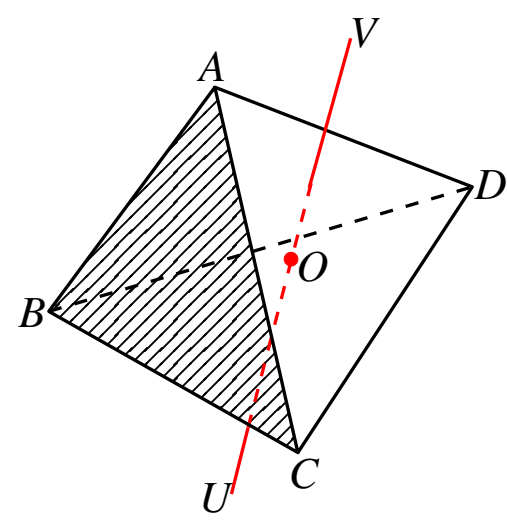

Figure 3: An illustration of the various geometric elements that were used to define the $R c_{\lambda}$ in 4-dimensions.

also lies on the dual edge $\lambda$. We refer to the tetrahedron formed by connecting $O$ with triangle hinge, $h=\{A, B, C\}$, as the restricted 3-volume, $\lambda_{h}^{*}$. There are four such reduced 3-volumes subdividing, $\lambda^{*}$. If we connect this shaded hinge, ${ }_{h}$, to edge $\lambda$, the resulting simplex, will be the $h \lambda$-reduced hybrid block of volume, $V_{\lambda h}=(1 / d) \lambda h_{\lambda}$.

The last two indices, $c$ and $b$, of the $R m$ in the far right-hand side of Eq. 35 identify the components of a circumnavigated area, and in our case this will be the Voronoi polygons, $h_{\mid \lambda}^{*}$, sharing edge $\lambda$. In the $h \lambda$-basis the two indices identifying this area element are $b=\lambda$ and $c=m_{h \lambda}$, or vise versa. As we argued earlier, the $R c$ tensor is associated with dual edge, $\lambda$, consequently $b=\lambda$ and $c=m_{h \lambda}$. The Ricci tensor is diagonal in the space of bivector-valued 2-forms. Therefore, since index $b=\lambda$ then index $a$ must be the moment arm $m_{\ell \lambda}$. There is a unique $m_{h \lambda}$ for each of the dual $\left(\begin{array}{l}d \\ 2\end{array}\right)$ Voronoi polygons, $h_{\mid \lambda}^{*}$, sharing edge $\lambda$. We define $R c_{\lambda}$ by summing over these $h \lambda$-moment arms. Therefore, we would sum over all all polygons, $h^{*}$, sharing edge, $\lambda$, i.e.

$$
\boldsymbol{R} c(\ldots, \boldsymbol{\lambda})=\boldsymbol{e}_{a} R_{c b}^{c a} \boldsymbol{e}^{b}\left(\boldsymbol{e}_{\lambda}\right)=\boldsymbol{e}_{a} R_{c \lambda}^{c a}=\sum_{h_{\mid \lambda}^{*}} \boldsymbol{e}_{a} R_{m_{\lambda} \lambda}^{m_{\lambda} a}
$$

Finally, since we showed that the simplicial $R m$ has compact support and uniformly distributed over the dual hybrid polytopes, $V_{(d-2)(d-2)^{*}}$, and since the geometry within this hybrid block is locally an Einstein space, then he only non-zero vector component, $a$ left in Eq. 42 is along $\lambda$. Therefore, the simplicial $R c_{\lambda}$ is diagonal,

$$
\boldsymbol{R} c(\ldots, \boldsymbol{\lambda})=\sum_{h_{\mid \lambda}^{*}} \boldsymbol{e}_{\lambda} R_{m_{\lambda} \lambda}^{m_{\lambda} \lambda}=\sum_{h_{\mid \lambda}^{*}} \boldsymbol{e}_{\lambda} R_{h^{*}}^{h^{*}}=\boldsymbol{e}_{\lambda} \sum_{h_{\mid \lambda}^{*}} R_{h^{*}}^{h^{*}}
$$

To complete the analysis of the right hand side of the SRF equation, we need also to define the Ricci tensor, $R c_{\ell}$, on an edge $\ell \in \mathcal{S}$. In other words we need to express this $\ell$-based Ricci tensor associating it with an appropriate weighted sum of the $\lambda$-based Ricci tensor equation (Eq. 40) onto the simplicial edge $\ell$. In essence, we trace over the components of $R c_{\lambda}$, one component for each $\lambda \in \partial \ell^{*}$, with respect to the simplicial edge $\ell$ [29]. We will have to similarly trace over the left-hand side of the SRF equation 
in Sec. 4.4 in order to prove the Corollary 1. In particular, consider an edge $\ell \in \mathcal{S}$ as illustrated in Fig. 4. Similarly, one integrates the $R c_{\ell}$ over the hybrid $d$-volume of the simplicial lattice associated with edge $V_{\ell}$ and equates this with the trace of the familiar hinge-based simplicial Ricci tensor $\left(R c_{h}\right)$ [34],

$$
R c_{\ell} V_{\ell}=\underbrace{\sum_{\lambda_{1 \ell^{*}}} R c_{\lambda} V_{\ell \lambda}}_{\begin{array}{c}
\text { simplicial form } \\
\text { of thetrace }
\end{array}}
$$

where the sum is over the dual edges, $\lambda$ bounding Voronoi polygon, $\ell^{*}$, and

$$
\begin{aligned}
V_{\ell} & =\left(\begin{array}{l}
d \\
1
\end{array}\right)^{-1} \ell \ell^{*}=\sum_{h_{\mid \ell}}\left(\begin{array}{l}
d \\
2
\end{array}\right)^{-1} h_{\ell} h^{*}, \text { and } \\
V_{\ell \lambda} & =V_{\ell \mid \lambda}=\sum_{h_{\mid \ell}}\left(\begin{array}{l}
d \\
2
\end{array}\right)^{-1} h_{\ell \mid \lambda} h^{*} .
\end{aligned}
$$

In order for us to obtain the right-hand side of Eq. 2 we make use of the following definition for the edge-based weighted average:

$$
\left\langle f_{h}\right\rangle_{\ell}:=\frac{1}{V_{\ell}} \sum_{h_{\mid \ell}} f_{h} V_{\ell h}=\frac{\sum_{h_{\mid \ell}} f_{h} h_{\ell}}{\sum_{h_{\mid \ell}} h_{\ell}} .
$$

This yields an explicit expression for the Ricci tensor, $R c_{\ell}$, associated to an edge, $\ell$, in the simplicial geometry,

$$
\begin{aligned}
R c_{\ell} & =\frac{1}{V_{\ell}} \sum_{\lambda_{\mid \ell^{*}}} R c_{\lambda} V_{\ell \lambda} \\
& =\frac{1}{V_{\ell}} \sum_{\lambda_{\mid \ell^{*}}}\left(\frac{1}{V_{\lambda}} \sum_{h_{\mid \lambda^{*}}} R c_{h}^{(h y b)} V_{\lambda h}\right) V_{\ell \lambda} \\
& =\sum_{\lambda_{\mid \ell^{*}}} \sum_{h_{\mid \lambda^{*}}} R c_{h}^{(h y b)}\left(\frac{V_{\lambda h}}{V_{\lambda}}\right)\left(\frac{V_{\ell \lambda}}{V_{\ell}}\right),
\end{aligned}
$$

or equivalently,

$$
R c_{\ell}=(d-1)\left\langle\left\langle R m_{h}\right\rangle_{\lambda}\right\rangle_{\ell}
$$

In the left-most diagram in Fig. 4, we focus our attention on an edge $\ell=\overline{A B}$ in a 4-dimensional simplicial lattice, $\mathcal{S}$, and illustrate the hinges $h$ sharing this edge. In this particular case there are six such hinges, $h_{i}=\overline{A B H_{i}}$, for $i=1,2, \ldots, 6$. The orientation on edge $\vec{\ell}=\overrightarrow{A B}$ induces an orientation on each of the six triangles sharing the edge. In particular, the triangle is a bi-vector in $\mathcal{S}$, where $h_{i}=\left|h_{i}\right| \hat{h}=$ 

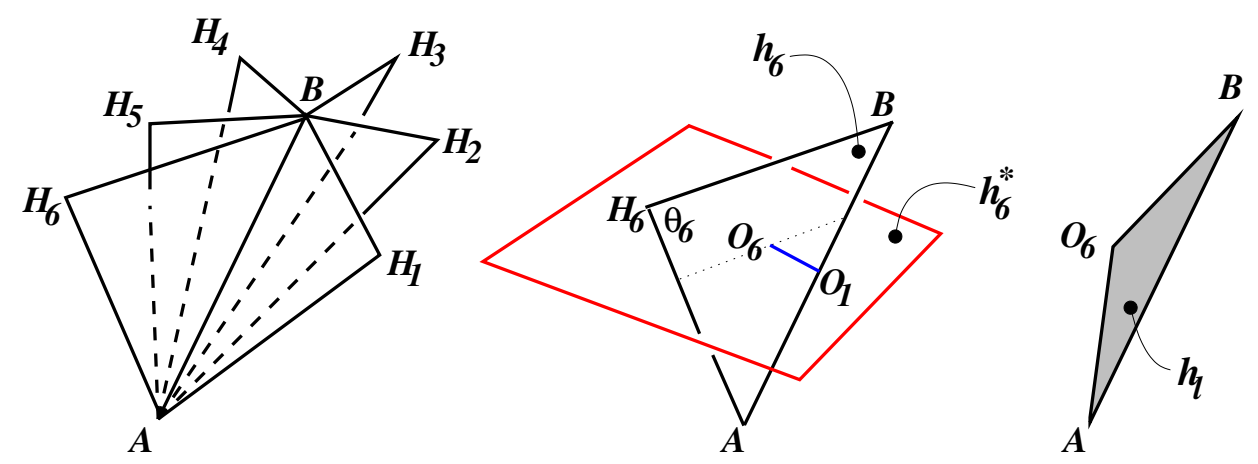

Figure 4: The geometric environs of an edge in a 4-dimensional simplicial lattice (left), one of its six hybrid blocks (center) and the edge-based hybrid area (right).

$\left|A B H_{i}\right|\left(\frac{\overrightarrow{A B} \wedge A \vec{H}_{i}}{2\left|A B N_{i}\right|}\right)$. In the central figure we focus our attention on one of the six hinges, $h_{6}$, sharing edge $\ell$, and its Voronoi dual polygon $h_{6}^{*} \in \mathcal{S}^{*}$. This dual polygon, $h_{6}^{*}$ is perpendicular to the hinge, $h_{6}$. The dotted line shown in the figure is simply the intersection of these orthogonal 2-dimensional surfaces. The orientation of the edge, $\ell$ also induces an orientation on the bi-vector, $h_{6}^{*} \in \mathcal{S}^{*}$. In particular, $\boldsymbol{h}_{i}^{*}=\left|h_{i}^{*}\right| \hat{\boldsymbol{h}}_{i}^{*}=$ $h_{i}^{*}\left(\hat{\boldsymbol{h}}_{i}\right)^{*}=h_{i}^{*}\left(\frac{\overrightarrow{A B \wedge} \overrightarrow{A H_{i}}}{2\left|A B N_{i}\right|}\right)^{*}$. The circumcenter of hinge $h_{6}$ is labeled $\mathcal{O}_{6}$. This point is equidistant from vertices $A, B$ and $H_{6}$. The " $h \lambda$-moment arm" is the edge $m_{h \lambda}=$ $\overline{O_{1} O_{6}}=(1 / 2) \vec{\ell} \cot (\theta)$ reaching from the center of edge $\ell$ to the circumcenter of triangle $h_{6}$. It is instrumental in the Cartan construction of the Regge-Einstein tensor [33]. The right-most figure shows the fraction of hinge $h_{6}$ closer to edge $\ell$ than to its other two edges. This edge-based triangle in $\mathcal{S}$ has area $h_{\ell}=\overline{A B O_{6}}=1 / 2 \ell^{2} \cot \left(\theta_{6}\right)$, which is useful in defining the $R c$ in $\mathrm{RC}$.

\subsection{The Simplicial Ricci Flow Equation}

Definition 1. We define the dual-edge Regge-Ricci flow equation for any compact, piecewise-flat simplicial geometry, $\mathcal{S}$, as an equation for each edge, $\lambda$, in the circumcentric dual lattice, $\mathcal{S}^{*}$,

$$
\left(\begin{array}{c}
\text { Fractional rate of change } \\
\text { of a circumcentric } \\
\text { dual edge, } \\
\frac{1}{\lambda} \frac{\partial \lambda}{\partial t}
\end{array}\right)=-\left(\begin{array}{c}
\text { Ricci tensor associated } \\
\text { to the circumcentric } \\
\text { dual edge, } \\
R c_{\lambda}
\end{array}\right)
$$

Remark 1. We have shown here and in [36] that the Ricci tensor is naturally associated with a dual edge, $\lambda \in \mathcal{S}^{*}$. We consider this edge, $\lambda$, and the associated hybrid cell, $V_{\lambda \lambda^{*}}$. We also use the $\ell \lambda$-hybrid orthogonal basis introduced in Sec. ??. Additionally, Sec. ?? and [36] showed that the mixed Ricci tensor is diagonal in $\lambda$. Motivated by Hamilton's RF equation, Eq. 9, with mixed indices in an orthogonal basis, then the RRF equation associated with this dual edge is,

$$
\frac{\dot{g}_{\lambda \lambda}}{g_{\lambda \lambda}}=-2 R_{\lambda}^{\lambda}=-2 R c_{\lambda} .
$$


Using the $g_{\lambda \lambda}$ component in the covariant metric for the $\ell \lambda$-hybrid basis, Eq. 27, we obtain the dual-edge RRF equation for $\lambda$,

$$
\frac{\dot{\lambda}}{\lambda}=-R c_{\lambda} .
$$

Since the geometry of $\mathcal{S}$ and $\mathcal{S}^{*}$ is completely determined by the edges, $\left\{\ell_{i}\right\} \in \mathcal{S}$, and nothing more, and since there are ordinarily many more dual edges, $\lambda$, than there are simplicial edges, $\ell$, then the system of equations given by the dual-edge RRF equation, Eq. 54, will ordinarily be overdetermined and have no solution. In other words each $\lambda$ is a function of the $\ell$ 's. Therefore, one solution would be for us to project, or trace, these dual-edge RRF equation onto the simplicial edges. This can be done using an appropriate weighted average.

Corollary 1. The simplicial Regge-Ricci flow equation for any compact, piecewiseflat simplicial geometry, $\mathcal{S}$, can be expressed as an equation for each edge, $\ell \in \mathcal{S}$,

$$
\left(\begin{array}{c}
\text { The volume averaged } \\
\text { fractional rate of change } \\
\text { of the dual edge, } \\
\left\langle\frac{1}{\lambda} \frac{\partial \lambda}{\partial t}\right\rangle_{\ell}
\end{array}\right)=-\left(\begin{array}{c}
\text { Ricci tensor associated to } \\
\text { the simplicial edge, } \\
R c_{\ell}
\end{array}\right) \text {. }
$$

Proof. We express the RRF equation by mapping the dual-edge RRF equation (Eq. 54) onto an edge $\ell$,

$$
\langle\dot{\lambda} / \lambda\rangle_{\ell}=-\left\langle R c_{\lambda}\right\rangle_{\ell}
$$

It suffices then to consider the right-hand side of this equation. Using the trace in $[29,34,35]$ on $R c_{\lambda}$, we note that

$$
R c_{\ell} V_{\ell}=\sum_{\lambda_{\mid \ell^{*}}} R c_{\lambda} V_{\ell \lambda}
$$

We also note that the $\ell-\lambda$ reduced hybrid volume is symmetric in its indices,

$$
V_{\ell \lambda}=V_{\lambda \ell}
$$

and we can expand the $\ell$ hybrid volume into a sum of these reduced hybrid cells,

$$
V_{\ell}=\sum_{\lambda_{\mid \ell^{*}}} V_{\ell \lambda}
$$

Therefore, we see by the definition of the weighted average in Eq. 57 that the righthand side of Eq. 56 is simply

$$
\left\langle R c_{\lambda}\right\rangle_{\ell}=R c_{\ell}
$$

and is given explicitly by Eq. 51. Applying the edge-based weighted average to the LHS of the equation as we just did for the RHS of the equation yields the simplicial RRF equation,

$$
\left\langle\frac{1}{\lambda} \frac{\partial \lambda}{\partial t}\right\rangle_{\ell}=-R c_{\ell}
$$




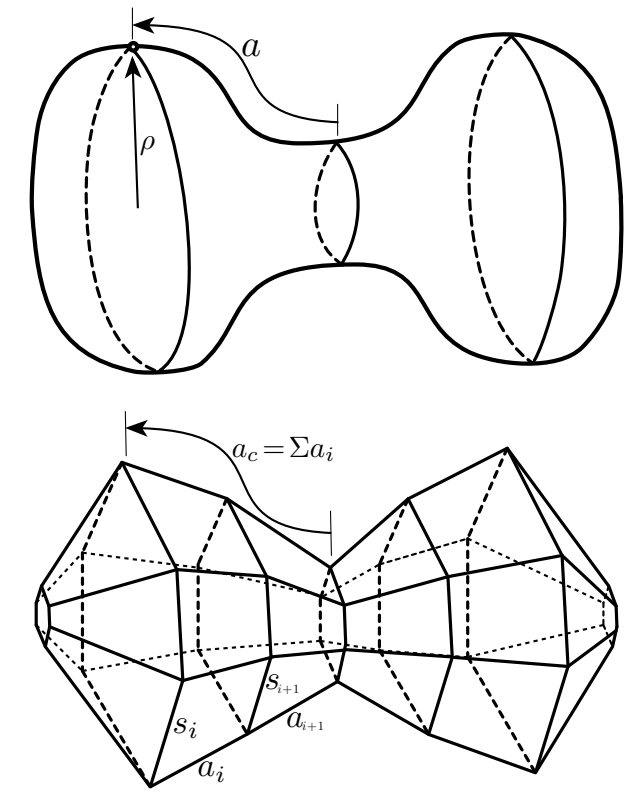

Figure 5: A 2-dimensional rendering of the simplicial double-lobed model.

\subsection{Benchmarking the SRF equations on 3-manifolds}

Under this effort we benchmarked the SRF equations on four models, the 3-cylinder, the 3-sphere, the anisotropic Bianchi IX geometry and the axisymmetric neck-pinch 3-geometry of Angenent and Knopf. These were reported in [8, 9, 10], respectively. The most important observation from these examples was the sensitivity of the SRF equations to the lattice properties. We observed stiff equations, and unless we remeshed the neck-pinch geometry along cubic splines, we were not able to evolve the geometry to a singularity. We observed that the SRF equations depended on the wellcenterdness of the circumcentric lattice.

We consulted with leading numerical scientists working with unstructured meshes, and were told that such sensitivity is not unexpected and that there are sophisticated re-meshing and mesh refinement methods that can handle such difficulties. We are currently collaborating with faculty at the University of Texas in the Department of Mathematics as well as in their Center for Numerical Analysis and Institute for Computational Science.

Most recently we took the continuum limit of the SRF equations for a sequence of finer and finer resolved lattice geometries modeling the neck-pinch of Angenent and Knopf. In Fig. 5 we illustrate a 2-dimensional rendering of the simplicial double-lobed model. For visualization purposes, we have suppressed one of the azimuthal angles for each of the 2-sphere cross sections. In two dimensions the piecewise linear (PL) surface tiles are trapezoids, where in 3-dimensions they are frustum polyhedra [10]. In this paper we take the limit of an ever more finely discretized sphere, and with an ever increasing number of spherical cross sections. In this limit we show that the 
continuum Hamiltonian RF equations are recovered from the SRF equations.

The initial data is determined by a radial profile function at $t=0$ for the double-lobed geometry, and amounts to specifying a function relating the cylindrical radius, $\rho$, to a scaled proper axial distance along the double-lobed geometry away from an equator or neck, $a \in\left\{a_{\min }, a_{\max }\right\}$,

$$
s=\psi(a) .
$$

By way of an example, if the double-lobed geometry has no neck, and were just a sphere of radius, $R_{0}$, this initial radial profile function is simply the cylindrical coordinate radius,

$$
\rho(a, t=0)=R_{0} \cos (a)
$$

We provide a simplicial approximation of an axisymmetric warped-product geometry at time $t$ characterized by an arbitrary $C^{2}$ radial profile

$$
\rho(a, t)=\rho(a) \forall a \in\left\{a_{\min }, a_{\max }\right\} .
$$

We first identify an arbitrarily large number $\left(N_{s} \rightarrow \infty\right)$ of nearly equal-spaced spherical cross sections. Next we examine one of these spheres, namely the $i^{\prime} t h$ crosssectional sphere. There are many ways to approximate this by a polyhedron with an arbitrarily large amount of vertices, $N_{s} \rightarrow \infty$. We utilize the symmetry of our model to concentrate only in the vicinity of a single point $\mathcal{O}$ on the sphere. At this point we project a infinitesimal flat-space hexagonal lattice onto its surface, as shown in Fig. 6. Here we take the length of the isosceles triangles that we are projecting onto the sphere of radius $\rho$ to be arbitrarily small, $\ell_{k} \ll \rho$. This yields an infinitesimal parameter in our model that we will drive to zero,

$$
\xi:=\frac{\ell}{\rho} \rightarrow 0
$$

In this manuscript we assume $\xi=\ell / r$ is a global infinitesimal for each of the, $N_{a}$, triangulated sphere cross sections in this model. In order to construct the SRF equations at $\mathcal{O}$ it is necessary for us to extend the lattice radially one more level out from the first six equilateral triangles so that we can examine 18 additional equilateral triangles, each of edge length $\ell_{k} \ll \rho$, that we project onto the surface of the sphere as shown in the right-hand side of Fig. 6 . The projected triangles will no longer be equilateral. In particular there will be two sets of 6 isosceles triangles, $\{\mathcal{O}, \mathcal{X}, \mathcal{Y}\}$ and $\{\mathcal{V}, \mathcal{X}, \mathcal{Y}\}$, as well as twelve triangles with three different edge lengths $\{\mathcal{X}, \mathcal{U}, \mathcal{V}\}$. 

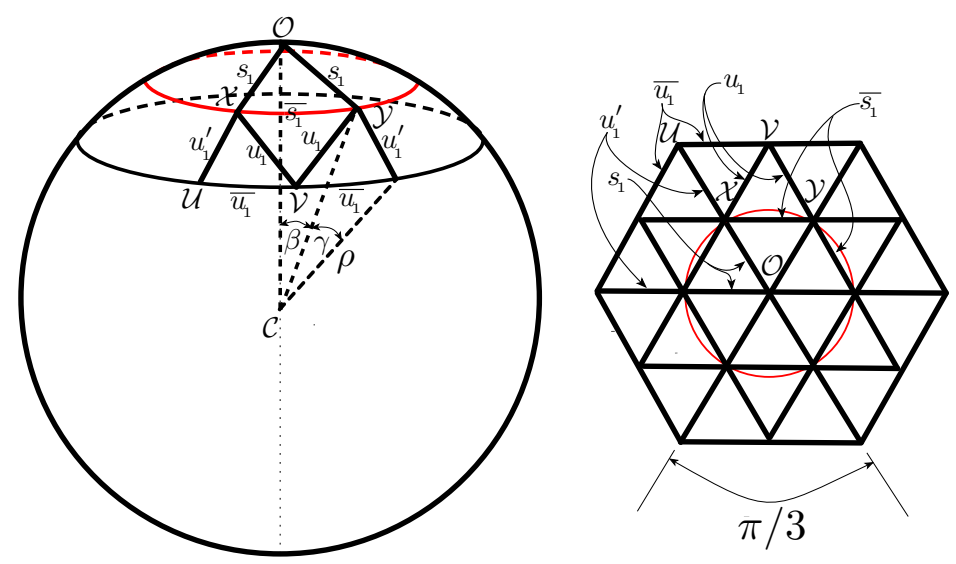

Figure 6: The 2-dimensional triangulated region around the vertex, $\mathcal{O}$, on a spherical cross-section of the axisymmetric geometry.

These 24 triangles are composed of combinations of six distinct edges,

$$
\begin{aligned}
& s=\cos ^{-1}\left(\frac{\overrightarrow{\mathcal{C O}}}{|\mathcal{C O}|} \cdot \frac{\overrightarrow{\mathcal{C X}}}{|\mathcal{C X}|}\right) \approx \rho \xi\left(1-\frac{1}{3} \xi^{2}+\frac{1}{5} \xi^{4}+O[\xi]^{6}\right), \\
& \bar{s}=\cos ^{-1}(\widehat{\mathcal{C X}} \cdot \widehat{\mathcal{C Y}}) \approx \rho \xi\left(1-\frac{11}{24} \xi^{2}+\frac{203}{640} \xi^{4}+O[\xi]^{6}\right) \text {, } \\
& u=\cos ^{-1}(\widehat{\mathcal{C X}} \cdot \widehat{\mathcal{C V}}) \approx \rho \xi\left(1-\frac{35}{24} \xi^{2}+\frac{1183}{640} \xi^{5} 4+O[\xi]^{6}\right) \text {, } \\
& \bar{u}=\cos ^{-1}(\widehat{\mathcal{C U}} \cdot \widehat{\mathcal{C V}}) \approx \rho \xi\left(1-\frac{11}{6} \xi^{2}+\frac{203}{40} \xi^{4}+O[\xi]^{6}\right), \\
& u^{\prime}=\cos ^{-1}(\widehat{\mathcal{C X}} \cdot \widehat{\mathcal{C U}}) \approx \rho \xi\left(1-\frac{7}{3} \xi^{2}+\frac{31}{5} \xi^{4}+O[\xi]^{6}\right) .
\end{aligned}
$$

We were able to explicitly show that the SRF equations converged to the continuum limit in second order. In particular we showed that for a small parameter $\xi$ shown in Fig. 6 the axial and radial SRF equations reproduced Hamilton's RF equations exactly, where we immediately recognized the right-hand side of the axial-edge SRF equation is the second derivative of $\rho$ with respect to $a$, and therefore we recover the corresponding continuum RF equation for the axial edge in the limit, namely

$$
\underbrace{\frac{\dot{a}_{i}}{a_{i}}=2 \frac{\left(\frac{\rho_{i+1}-\rho_{i}}{a_{i}}\right)-\left(\frac{\rho_{i}-\rho_{i-1}}{a_{i}}\right)}{a_{i}}}_{\text {continuum-limit SRF equation }} \Longrightarrow \underbrace{\frac{\dot{a}}{a}=2 \frac{\rho^{\prime \prime}}{\rho}}_{\text {continuum RF equation }} .
$$

We also examined the $\sigma_{i}$-edge SRF equation and showed it converges to the continuum RF equation to second order in $\xi$. In particular, we recover the corresponding 
continuum RF equation for the axial edge in the continuum limit, namely

$$
\underbrace{\frac{\dot{\rho}_{i}}{\rho_{i}}=\frac{\frac{\left(\frac{\rho_{i+1}-\rho_{i}}{a_{i}}\right)-\left(\frac{\rho_{i}-\rho_{i-1}}{a_{i}}\right)}{a_{i}}}{\rho_{i}}+\frac{\left(\frac{\rho_{i+1}-\rho_{i}}{a_{i}}\right)^{2}}{\rho_{i}^{2}}-\frac{1}{\rho_{i}^{2}}}_{\text {continuum-limit SRF equation }} \Longrightarrow \underbrace{\frac{\dot{\rho}}{\rho}=\rho^{\prime \prime} / \rho+\left(\rho^{\prime} / \rho\right)^{2}-1 / \rho^{2}}_{\text {continuum RF equation }} .
$$

The errors in these equations were of second order in our small parameter, for example the Gaussian curvature at edge $\hat{a}_{i}$ is

$$
K_{\hat{a}_{i}}:=\frac{\epsilon_{\hat{a}_{i}}}{\hat{a}_{i}^{*}} \approx-\frac{4\left(\rho_{i}-\rho_{i+1}\right)^{2}}{a_{i}^{2}\left(\rho_{i}+\rho_{i+1}\right)^{2}}\left(1-\frac{5\left(\rho_{i+1}-\rho_{i}\right)^{2}}{24 a_{i}^{2}} \xi^{2}+O\left(\xi^{4}\right)\right),
$$

and shows the errror term in $\xi^{2}$. Other terms in the SRF equations showed similar convergence without further complications. This is a rather strong result supporting our definitions of the curvature and our SRF equation definition. More general convergence calculations could be explored.

\subsection{SRF: Singularities, Surgery, Stability and the Simplicial Diffeomorphisms}

In this final report we have formulated a discrete form of the Hamilton RF equations for a piecewise flat simplicial geometry for any dimension, $d$. We imagine that this simplicial formulation of RF will provide new insights both numerically and, more importantly, in representing the singularity and soliton structure on piecewise flat simplicial lattices. It may be possible to use the RRF mathematics to explore singularity structure in three and higher dimensions in novel ways [46]. Perhaps there are minimal simplicial structures characterizing RF singularities of various types, in other words we can ask, "Given a RF singularity (Type-1, Type-2 etc.), what is the simplest simplicial lattice that exhibits this singularity?"

Hamilton demonstrated that the RF equations will, under certain conditions, evolve to produce singularities [47]. He discussed some intuitive solutions for dumbbell geometries producing pinching singularities. For the RRF equations to produce a faithful representation of Hamilton's RF, the lattice geometry will need to evolve so as to properly approach these pinching singularities and then to avoid them by surgery $[6,47]$. The analysis of such singularities using the RRF equations is one of the primary directions guiding our current research. We are in the process of analyzing the dumbbell model with 3-sphere topology to reproduce the recent results of $[11,12]$ using the RRF equations. Our preliminary results demonstrate neck pinching, although more analysis is necessary [45]. Once we demonstrate that the RRF equations reproduce quantitatively correct neck pinch singularity formation we will begin to examine a simplicial implementation of surgery. This will involve separating the neck pinch into two separate lattices and then generate an appropriate simplicial cap for each of these, thereby continuing the RRF evolution to produce a connected sum of geometries. While we are only beginning in this direction, we believe it possible, at least in 3-dimensions, to produce an automated surgery procedure for a lattice geometry in order to produce 
a complete RF evolution to a connected sum of Thurston-type geometries for a broad range of initial simplicial geometries and topologies [7].

We have begun a numerical implementation of the RRF equations in 3-dimensions on 3-sphere lattices [15]. In particular, we are currently analyzing the RRF of geometries with 3-sphere topology with everywhere positive Ricci curvature [15]. In this analysis, the initial simplicial geometry is a perturbation of the length of each of the 720 edges of the 600-cell regular polytope about their regular values so as to keep the $R c$ positive everywhere. Hamilton proved that non-uniformities in such geometries will converge to the regular 600-cell polytope exponentially fast [30] provided that the RRF faithfully reproduces continuum RF. We are also examining Type- 1 and Type -2 singularity formation in a simplicial representation of the dumbbell model $[45,48]$. Our preliminary results for both models are qualitatively consistent uniformization; however, we need to carefully examine the detailed exponential fast uniformization predicted by Hamilton and to examine numerical behavior of the normalized RRF equations and their stability/instability. For small perturbation around the 600-cell model we examined the 720 eigenvalues of the Jacobian for the RRF equations and we find numerous positive real values indicating the well-known instability of the linearized RF equations about a 3-sphere geometry [15]. Examining the stability of the RRF equations and providing suitable reformulations of these equations is the primary focus of our research.

We are animated by three directions for this analysis. First, Perelman introduced a gauge fixed (conformal and diffeomorphism) modified RF evolution by introducing an entropy integral - an integral which produces uniformization via a gradient flow [6]. We ask, "What is the simplicial analogue of this for a piecewise flat simplicial lattice"? Second, an approximate simplicial diffeomorphism structure for a piecewise flat simplicial geometry has been understood [49] and successfully implemented in RC. This should enable us to examine a modified RRF equation using these approximate diffeomorphic degrees of freedom in order to improve the uniformization of the simplicial geometry (e.g. well-centered Delaunay lattices, maximally regular simplexes, etc.). Finally, there are many similarities between RF and issues involved in evolving black hole spacetimes that we believe can inform both fields of research.

The numerical solution of Hamilton's RF equations in 3 and higher dimensions involve much the same issues as the vacuum black hole solutions of the Einstein equations. In fact, the Einstein equations for a vacuum spacetime is just the Ricci tensor. First, Hamilton showed that one can encounter various singularities in RF which is also true for the numerical evolution of black hole spacetimes. Secondly, our recent results suggest that the RRF equations have instabilities in certain cases $[15,45]$ as do the discretized version of the standard ADM equations in numerical relativity. Thirdly, Hamilton suggested a surgery procedure in order to integrate through the neck pinch singularities in much the same way as the moving puncture procedure or horizon excision procedure is used in numerical relativity to numerically evolve the spacetime geometry avoid the black hole singularity. Finally, in general relativity one utilizes the diffeomorphic degrees of freedom and conformal factors through the initial value data as well as a judicious choice of the lapse and shift functions during evolution so as 
to provide a stable solution of Einstein's equations that also avoids singularities (e.g. constant mean curvature slicing and minimal shear). In a similar vein, Perelman's entropy function utilizes diffeomorphic and conformal modes to provide a gradient flow for a modified RF equation. This suggests that experience gained in general relativity may inform similar issues in the numerical analysis of RF, just as experience with RF evolutions may offer insights into numerical relativity. We view this as an exciting avenue of research that may bridge two disciplines.

\subsection{Adiabatic Isometric Map: Applying SRF to Weyl's 1916 Isometric Embed- ding Theorem for Surfaces}

This project supported the dissertation research of two graduate students from FAU, one student from Stony Brook, and a postdoctoral fellow at Harvard University. One of the graduate students at FAU (Shannon Ray) and postdoctoral fellow (Jonathan McDonald) applied SRF to define quasi-local mass of Wang and Yau. This required a novel and efficient numerical implementation of the Weyl-Alexandrov isometric embedding problem. The resulting Adiabatic Iscometric Map (AIM) approach utilized SRF and has provided a robust solution to this problem [14].

Alexandrov proved that any simplicial complex homeomorphic to a sphere with strictly non-negative Gaussian curvature at each vertex can be isometrically embedded in $\mathbb{R}^{3}$ as a convex polyhedron [50]. Due to the nonconstructive nature of his proof, there have yet to be any algorithms that realizes the Alexandrov embedding in polynomial time. Following his proof, we produced the adiabatic isometric mapping (AIM) algorithm. Tests of AIM applied to two different polyhedral metrics indicate that its run time is sub cubic with respect to the number of vertices.

Under this research we developed the adiabatic isometric mapping (AIM) algorithm which is a numerical realization of Alexandrov's proof for embedding convex polyhedral metrics. Like D. Kane et. al [51] our algorithm produces approximately convex polyhedrons which we will show are adequate solutions to Alexandrov's embedding problem. Taking techniques from the algorithms mentioned, AIM begins by applying Ricci flow to a convex polyhedral metric. Next we embed the constant curvature surface and uses it as the starting point for an embedding flow similar to M. Jasiulek and M. Korzyński's [52]. To step from one conformally related polyhedron to the next, we use Newton's method to minimize an objective function based on edge lengths of the polyhedral metric and distances in $\mathbb{R}^{3}$. To avoid the problem of local minima found in Bondarescu et. al [53], we use the coordinates of the constant curvature polyhedron as the initial guess for Newton's method. This puts us close enough to the solution so that we quickly converge to the global minimum. We then use the newly embedded polyhedron as the initial value data for our next step. This is repeated until we reach the original polyhedral metric. We also show that if our steps between surfaces are small enough, we can reach a smooth polyhedron that adequately represents Alexandrov's polyhedron thus avoiding the problem of non-smooth solutions seen by H-P. Nollert and $\mathrm{H}$. Herold [54]. We found that AIM is capable of embedding with accuracy in the edge lengths up to double precision. It is also capable of embedding non-convex polyhedral metrics though we do not know the extent at which it is applicable. 
To navigate through the "sea of solutions" without resorting to constrained optimization, AIM uses a three step procedure, (1) uniformization via SRF that provides a dimensional reduction from 3 to 2 dimensions, (2) uniform surface embedding, and (3) a pull-back of the coordinates from the uniformed surface to the original surface via the backward adiabatic march (BAM). These three steps ameliorate many difficulties in solving the system of equations and provide controllable criteria for obtaining a suitable solution. We briefly describe these steps in the next three subections which are illustrated in Fig. 7.

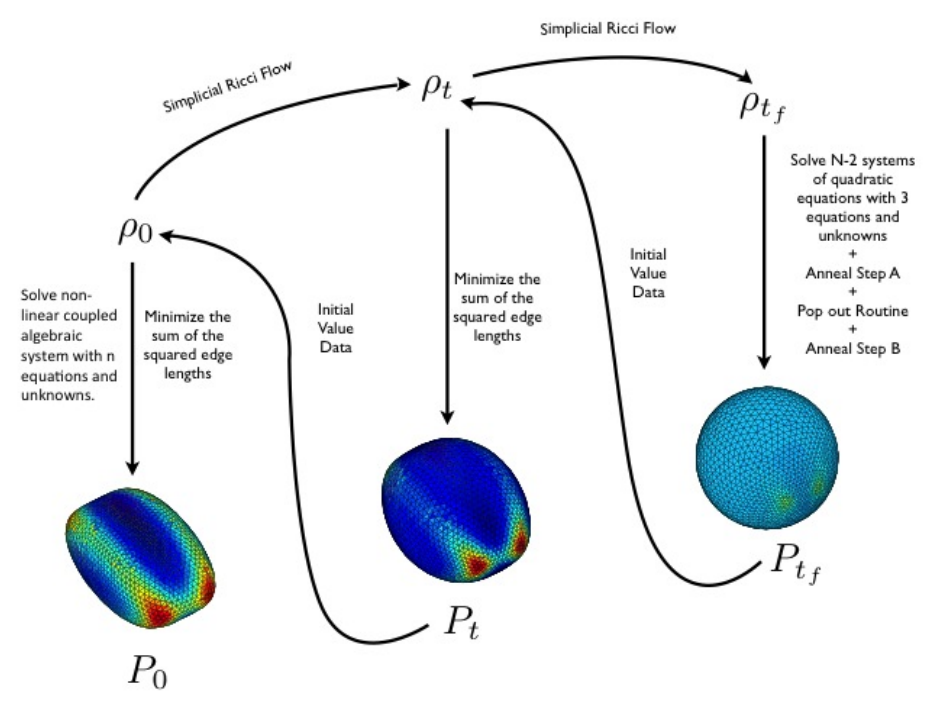

Figure 7: This figure visualizes the three steps of the AIM algorithm.

In Fig. 7 we illustrate the three steps of the AIM algorithm. The section entitled uniformization via Ricci flow begins at $\rho_{0}$ in the upper left corner and ends at $\rho_{t_{f}}$ in the upper right corner. The uniform surface embedding begins at $\rho_{t_{f}}$ and ends at the bottom right of the figure. Finally, the adiabatic backward march sections starts at $P_{t_{f}}$ in the lower right corner and ends at $P_{0}$ in the lower left corner of the figure.

We plan on using this to define quasi-local congestion on an embedded network geometry. We will borrow the theoretical construction of Wang and Yau in their definition of quasi-local mass in general relativity and apply this to the simplicial geometry of an embedded network. Needed in this construction is a Hamiltonian for the embedded network. Guiding this research is the widely accepted conjecture that curvature is a measure of congestion and load balance in networks. While we are just at the beginning of this research, we believe this holds promise to address one of the deepest questions in complex networks: "What is the normal state of the network?"

\section{CONCLUSIONS}

The work presented here proposes a novel approach to 3-manifold recognition. It gives us a natural path for transitioning this research beyond this one-year exploratory 
project to the following milestones:

1. Couple SRF with persistent homology to identify singularity formation.

2. Develop a fully-efficient algorithm using SRF with surgery and diffeomorphism to decompose simplicial 3-manifolds into a finite connected sum of their prime components.

3. Develop quasi-local congestion and use this in $\mathrm{PH}$ as a filtration parameter to identify, monitor and ameliorate abnormal states of a complex network.

4. Apply SRF to 3-geometries with boundary for applications extending domain of combinatorial RF on 2-surfaces.

\section{Acknowledgements}

Contractor acknowledges Government's support in the publication of this report. We acknowledge all co-authors and colleagues that contributed to this research. This material is based upon work funded by AFRL, under AFRL Contract No. FA8750-11-20089. Any opinions, findings and conclusions or recommendations expressed in this material are those of the author and do not necessarily reflect the views of AFRL. 


\section{REFERENCES}

[1] X. Yu, X. Yin, W. Han, J. Gao \& X. Gu, "Scalable routing in 3D high genus sensor networks using graph embedding," INFOCOM 2012: 2681-2685; Y. Wang, J. Shi, X. Yin, X. Gu, T. F. Chan, S-T Yau, A. W. Toga, P. M. Thompson, "Brain surface conformal parameterization with the Ricci flow," IEEE Trans. Med. Imaging 31(2) (2012) 251-264. X. Gu, F. Luo \& S-T Yau, "Fundamentals of computational conformal geometry," Mathematics in Computer Science 4(4) (2010) 389429; B. Chow \& F. Luo, "Combinatorial Ricci flows on surfaces," J. Differential Geometry 63 (2003) 97-129.

[2] H-D. Cao, B. Chow, S-C Chu \& S-T Yau, eds., Collected Papers on Ricci Flow in Series in Geometry and Topology, Volume 37 (International Press; Somerville, MA; 2003).

[3] B. Chow \& D. Knopf, The Ricci Flow: An Introduction, Mathematical Surveys and Monographs, Volume 110 (American Mathematical Society; Providence, RI; 2004).

[4] B Chow, P. Lu \& L. Ni, Hamilton's Ricci Flow, Graduate Studies in Mathematics, Volume 77 (American Mathematical Society; Providence, RI; 2006).

[5] B. Chow, S-C Chu, D. Glickenstein, C. Guenther, J. Isenberg, T. Ivey, D. Knopf, P. Lu, F. Luo \& L. Ni, The Ricci Flow: Techniques and Applications, Part 1: Geometric Aspects, Mathematical Surveys and Monographs, Volume 135 (American Mathematical Society; Providence, RI; 2007).

[6] G. Perelman, "The entropy formula for the Ricci flow and its geometric applications," preprint, math.DG/0211159; G. Perelman, "Ricci flow with surgery on three-manifolds," preprint, math.DG/0303109; \& G. Perelman, "Finite extinction time for the solutions to the Ricci flow on certain three-manifolds," preprint, math.DG/0307245.

[7] W. Thurston, Three-dimensional geometry and topology, Vol. 1. Edited by Silvio Levy, Princeton Mathematical Series, 35, (Princeton University Press, Princeton, NJ, 1997).

[8] W. A. Miller, J. R. McDonald, P. M. Alsing, D. X. Gu \& S-T Yau, "Simplicial Ricci Flow," Commun. Math. Phys. 329 579-608 (2014).

[9] Alsing, P. M., Miller, W. A., Corne, M., Gu, X. D., Lloyd, S., Ray, S. and Yau, S-T, "Simplicial Ricci Flow: An Example of a Neck Pinch Singularity in 3D," Geom., Imaging Comp. in press (2014) ; arXiv:1308.4148 [math.DG].

[10] W. A. Miller, P. Alsing, M. A. Corne \& S. Ray, "Equivalence of Simplicial Ricci Flow and Hamilton's Ricci Flow for 3D Neckpinch Geometries," Geom., Imaging and Comp. (2014) in press.

[11] S. Angenent \& D. Knopf, "An example of neckpinching for Ricci flow on $S^{n+1}$," Math. Res. Lett. 11 (2004) 493-518.

[12] S. Angenent, J. Isenberg and D. Knopf, "Formal matched asymptotics for degenerate Ricci flow neckpinches," Nonlinearity 24 (2011), 2265-2280. 
[13] Alsing. P., Blair, H. A., Corne, M., Jones, G., Miller, W. A., Mischaikow, K. and Nanda, V. "Topological Signatures of Singularities in Simplicial Ricci Flow," to be submitted to J. Comp. Geom. (2014).

[14] S. Ray, W. A. Miller, P. M. Alsing and S-T Yau, "Adiabatic Isometric Mapping Algorithm for Embedding Polyhedral Metrics in Euclidean 3 Space, to be submitted to Class. Quantum Grav. (2014).

[15] P. M. Alsing, M. Corne, J. R. McDonald, W. A. Miller, S. Ray, "3D simplicial Ricci flow of the 600-cell model," in preparation (2013).

[16] Y. Lin, G. Lippner and S-T Yau, "Quantum Tunneling on Graphs," Commun. Math. Phys. 311, 113-132 (2012).

[17] Yong Lin and Shing-Tung Yau, "Ricci curvature and eigenvalue estimate on locally finite graphs," Math. Res. Lett. 17 (2010) 343356.

[18] J. Peiro \& S. Sherwin, Finite Difference, Finite Element and Finite Volume Methods For Partial Differential Equations, in Handbook of Materials Modeling, Volume 1, Methods and Models, Springer, 2005.

[19] S. Humphries, Jr., Finite-Element Methods for Electromagnetism, http://www.fieldp.com/freeware/finite_element_electromagnetic.pdf; originally published as Field Solutions on Computers (ISBN 0-8493-1668-5) (Taylor and Francis, Boca Raton, 1997).

[20] T. Regge, "General relativity without coordinates," Il Nuovo Cimento 19 (1961) 558-571.

[21] A. P. Gentle, W. A. Miller, "A fully (3+1)-D Regge calculus model of the Kasner cosmology," Class.Quant.Grav. 15 (1998) 389-405.

[22] M. Desbrun, A. N. Hirani, M. Leok \& J. E. Marsden, "Discrete exterior calculus," e-print arXiv:math/0508341v2 [math.DG] on arxiv.org (2005).

[23] B. Chow \& F. Luo, "Combinatorial Ricci flows on surfaces," J. Diff. Geom. 63 (2003) 97-129.

[24] D. Glickenstein, "Discrete conformal variations and scalar curvature on piecewise flat two- and three-dimensional manifolds," J. Diff. Geom. 87 (2011) 201238.

[25] D. Glickenstein, "Geometric triangulations and discrete Laplacians on manifolds," arXiv:math/0508188 [math.MG].

[26] H. Ge, "Discrete Quasi-Einstein Metrics and Combinatorial Curvature Flows in 3-Dimension," arXiv:1301.3398 [math.DG].

[27] R. Forman, "Bochner's Method for Cell Complexes and Combinatorial Ricci Curvature," Discrete Comput. Geom. 29 (2003) 323374.

[28] D. Knopf, "Estimating the trace-free Ricci tensor in Ricci flow," Journal: Proc. Amer. Math. Soc. 137 (2009), 3099-3103.

[29] J. R. McDonald, W. A. Miller, P. M Alsing, X. D. Gu, X. Wang \& S-T Yau, "On exterior calculus and curvature in piecewise-flat manifolds," paper submitted to J. Math. Phys. (2012) arxiv.org/abs/1212.0919. 
[30] R. Hamilton, “Three-manifolds with positive Ricci curvature," J. Diff. Geom 17 (1982), 255-306.

[31] E. Cartan, Leogns sur la Géométrie des Espaces de Riemann (Gauthier-Villars; Paris, 1951); translated into English by J. Glazebrook, Geometry of Riemann Spaces, Lie Groups: History Frontiers and Applications XIII (Math Science Press, Brookline, MA, 1983).

[32] R. Friedberg and T. D. Lee, Nucl. Phys. B242 (1984) 145-166.

[33] W. A. Miller, "The geometric content of the Regge equations as illuminated by the boundary of a boundary principle," Found. Phys. 16 (1986) 143-169.

[34] W. A. Miller, "The Hilbert action in Regge calculus," Class. Quantum Grav. 14 (1997) L199-L204.

[35] J. R. McDonald \& W. A. Miller, “The scalar curvature in Regge calculus," Class. Quantum Grav. 25 (2008) 195017.

[36] P. M. Alsing, J. R. McDonald \& W. A. Miller, "The simplicial Ricci tensor," Class. Quantum Grav. 28 (2011) 155007 (17 pp).

[37] E. Vanderzee, A. N. Hirani, D. Guoy \& E. A. Ramos, arXiv:0802.2108v3 [cs.CG] 18 Aug 2009.

[38] M. Caselle, A. D’Adda \& L. Magena, Phys. Lett. B232 (1989) 457.

[39] J. R. McDonald, W. A. Miller, “A discrete representation of Einstein's geometric theory of gravitation: the fundamental role of dual tessellations in Regge calculus," submitted to "Tessellations in the Science: Virtues, Techniques and Applications of Geometric Tilings," ed. R. van de Weijgaert, G. Vegter, J. Ritzerveld and V. Icke (2008); arXiv:0804.0279

[40] J. B. Hartle \& R. Sorkin, "Boundary terms in the action for the Regge calculus," General Relativity and Gravitation, 13, no. 6 (1981) 541-549.

[41] R. Sorkin, "Time Evolution Problem in Regge Calculus." Phys. Rev. D12 (1975) 385-396.

[42] L. P. Eisenhart, Riemannian Geometry, $8^{t} h$ edition (Princeton Univ. Press, Princeton, NY, 1997).

[43] R. Friedberg \& T. D. Lee, "Derivation of Regge's action from Einstein's theory of general relativity," Nuclear Physics B242 (1984) 145166.

[44] H. S. M. Coxeter, Regular Polytopes, (Dover Publ., NY, 1973).

[45] P. M. Alsing, J. R. McDonald, W. A. Miller, C. Tison \& X. Wang, "3D simplicial Ricci flow of the dumbbell model," in preparation (2013).

[46] H.-D. Cao, Private communication, UBC, Vancouver BC (2011).

[47] R. S. Hamilton, "The formation of singularities in the Ricci flow," Surveys in Differential Geometry 2 (1995) 7-136.

[48] X. Gu \& W. Zeng, Private communication, SUNY Stoney Brook (2012). 
[49] A. P. Gentle, A. Kheyfets, J. R. McDonald \& W. A. Miller, "A Kirchhoff-like conservation law in Regge calculus," Class. Quant. Grav. 26 (2009) 015005.

[50] A. D. Alexandrov, Intrinsic Geometry of Convex Surfaces, (Classics of Soviet Mathematics; CRC Press; 1 ed., 2006).

[51] D. Kane, G. N. Price, and E. D. Demaine, "A pseudopolynomial algorithm for Alexandrovs theorem," Lecture Notes in Computer Science 5664435446 (2009).

[52] M Jasiulek and M Korzyn?ski, "Isometric embeddings of 2-spheres by embedding flow for applications in numerical relativity," Class. Quantum Grav. 29 no. 15,155010 (2012).

[53] M. Bondarescu, M. Alcubierre, and E. Seidel, "Isometric embeddings of blackhole horizons in three-dimensional flat space," Class. Quantum Grav. 19 no. 2, 375 (2002).

[54] H. Nollert and H. Herold, "Visualization in curved spacetimes: visualization of surfaces via embedding," in Relativity and scientific computing, computer algebra, numerics, visualization F. W. Hehl, R. A. Puntigam, and H. Ruder, eds, pages 330351 (Springer; 1996). 


\section{LIST OF SYMBOLS, ABBREVIATIONS AND ACRONYMS}

$\begin{array}{ll}\text { AIM } & \text { Adiabatic Isometric Map } \\ \text { AFRL } & \text { Air Force Research Laboratory } \\ \text { BAM } & \text { Backward Adiabatic March } \\ \text { CRF } & \text { Combinatorial Ricci Flow } \\ \text { DEC } & \text { Discrete Exterior Calculus } \\ \text { FAU } & \text { Florida Atlantic University } \\ \text { GT } & \text { Geometrization Theorem } \\ \text { INFOCOM } & \text { IEEE Conference on Computer Communications } \\ \text { PH } & \text { Persistent Homology } \\ \text { PI } & \text { Principle Investigator } \\ \text { PL } & \text { Piecewise Linear } \\ \text { RC } & \text { Regge Calculus } \\ \text { RF } & \text { Ricci Flow } \\ \text { RI } & \text { Information Directorate } \\ \text { RITC } & \text { Emerging Computing Technology } \\ \text { SRF } & \text { Simplicial Ricci Flow } \\ \text { SUNY } & \text { State University of New York } \\ \text { UBC } & \text { University of British Columbia } \\ \text { UG } & \text { Undergraduate } \\ \text { US } & \text { United States } \\ \text { UT } & \text { Uniformization Theorem }\end{array}$

